\title{
Synapse alterations precede neuronal damage and storage pathology in a human cerebral organoid model of CLN3-juvenile neuronal ceroid lipofuscinosis
}

Gemma Gomez-Giro 1,2, Jonathan Arias-Fuenzalida', Javier Jarazo', Dagmar Zeuschner ${ }^{3}$, Muhammad Ali, ${ }^{4,5}$ Nina Possemis ${ }^{1}$, Silvia Bolognin', Rashi Halder ${ }^{6}$, Christian Jäger ${ }^{7}$, Willemijn F. E. Kuper ${ }^{8}$, Peter M. van Hasselt ${ }^{8}$, Holm Zaehres ${ }^{2,9}$, Antonio del Sol ${ }^{4,10,11}$, Herman van der Putten ${ }^{12}$, Hans R. Schöler ${ }^{2}$ and Jens C. Schwamborn ${ }^{1 *}$ (D)

\begin{abstract}
The juvenile form of neuronal ceroid Lipofuscinosis (JNCL) is the most common form within this group of rare lysosomal storage disorders, causing pediatric neurodegeneration. The genetic disorder, which is caused by recessive mutations affecting the CLN3 gene, features progressive vision loss, cognitive and motor decline and other psychiatric conditions, seizure episodes, leading to premature death. Animal models have traditionally aid the understanding of the disease mechanisms and pathology and are very relevant for biomarker research and therapeutic testing. Nevertheless, there is a need for establishing reliable and predictive human cellular models to study the disease. Since patient material, particularly from children, is scarce and difficult to obtain, we generated an engineered a CLN3-mutant isogenic human induced pluripotent stem cell (hiPSC) line carrying the c.1054C $\rightarrow$ T pathologic variant, using state of the art CRISPR/Cas9 technology. To prove the suitability of the isogenic pair to model JNCL, we screened for disease-specific phenotypes in non-neuronal two-dimensional cell culture models as well as in cerebral brain organoids. Our data demonstrates that the sole introduction of the pathogenic variant gives rise to classical hallmarks of JNCL in vitro. Additionally, we discovered an alteration of the splicing caused by this particular mutation. Next, we derived cerebral organoids and used them as a neurodevelopmental model to study the particular effects of the $\mathrm{CLN} 3^{\mathrm{Q} 352 \mathrm{X}}$ mutation during brain formation in the disease context. About half of the mutation -carrying cerebral organoids completely failed to develop normally. The other half, which escaped this severe defect were used for the analysis of more subtle alterations. In these escapers, whole-transcriptome analysis demonstrated early disease signatures, affecting pathways related to development, corticogenesis and synapses. Complementary metabolomics analysis confirmed decreased levels of cerebral tissue metabolites, some particularly relevant for synapse formation and neurotransmission, such as gamma-amino butyric acid (GABA). Our data suggests that a mutation in CLN3 severely affects brain development. Furthermore, before disease onset, disease -associated neurodevelopmental changes, particular concerning synapse formation and function, occur.
\end{abstract}

Keywords: JNCL, CLN3 disease, CRISPR/Cas9, Cerebral organoids, Neurodevelopment, Synapses

\footnotetext{
* Correspondence: jens.schwamborn@uni.lu

${ }^{1}$ Luxembourg Centre for Systems Biomedicine (LCSB), Laboratory of

Developmental and Cellular Biology, University of Luxembourg, Avenue du

Swing 6, Belvaux, Luxembourg

Full list of author information is available at the end of the article
}

(c) The Author(s). 2019 Open Access This article is distributed under the terms of the Creative Commons Attribution 4.0 International License (http://creativecommons.org/licenses/by/4.0/), which permits unrestricted use, distribution, and

reproduction in any medium, provided you give appropriate credit to the original author(s) and the source, provide a link to the Creative Commons license, and indicate if changes were made. The Creative Commons Public Domain Dedication waiver (http://creativecommons.org/publicdomain/zero/1.0/) applies to the data made available in this article, unless otherwise stated. 


\section{Introduction}

Juvenile neuronal ceroid lipofuscinosis (JNCL), also commonly referred to as Batten disease or CLN3 disease (OMIM \#204200) belongs to the neuronal ceroid lipofuscinoses: a larger group of lysosomal storage disorders which represent a major cause of neurodegeneration in children and young adults $[6,81]$. With an estimated incidence range of $0.2-7$ per 100,000 births worldwide, and an increased prevalence in northern European populations $[38,76]$, JNCL is considered a rare disease. Its symptoms typically manifest between 4 and 8 years of age with a rapid and progressive vision loss [59]. The disease advances with cognitive [42] and motor decline [43], accompanied by seizure episodes and behavioural changes, and inevitable leads to premature death during the second or the third decade of life, in the classical disease phenotype [71]. In addition to neurodegeneration, neurodevelopment may also be affected. Although animal models have broadened our knowledge about disease mechanisms, protein localization, function and interactions, the neurodevelopmental component to JNCL is still poorly understood. Only a few studies were able to show to some extent developmental abnormalities in newborn mice [58] or zebra fish embryos and larvae [78]. However, they might not recapitulate the features of the human disease.

JNCL is caused by recessively inherited mutations in the CLN3 gene [33]. Up to date, a total number of 67 different mutations occurring in the CLN3 gene have been compiled by the NCL Mutation and Patient Database (http://www.ucl.ac.uk/ncl/CLN3mutationtable.htm). Whereas most of the JNCL patients (80-85\%) are homozygous for a $1.02 \mathrm{~kb}$ deletion of exons 7 and 8, compound heterozygous cases or homozygous for the different single nucleotide variants are scarce and usually manifest in one or few families [41]. The existence of CLN3 missense mutations that cause other disorders emphasises the need to study these variants closely [79]. Initial studies using patient-specific human induced pluripotent stem cells (hiPSCs) showing in vitro effects of CLN3 mutations on the endocytic pathway and calcium homeostasis and autophagy have been published [18, 49]. However, patient-derived hiPSCs have the drawback that, aside from the disease-associated mutations, they carry the genetic background of the affected individuals, which can be extremely diverse between patients, making associating phenotypes directly to a particular gene mutation a complicated task. To overcome these limitations, we used state of the art CRISPR/Cas9 genome editing technologies [4] and introduced a disease-causing mutation into the CLN3 gene of healthy hiPSCs. The newly generated isogenic pair represents an advantage compared to gene corrected cell lines [83], as it allows to study the contribution of a particular mutation to the disease phenotype, without any concomitant effect of the patient's genetic background.

In this study, we used cerebral organoids as model for early brain development $[45,51]$ to investigate whether CLN3 deficiency affects fundamental neurodevelopmental mechanisms, such as growth and differentiation. Our results highlight transcriptional and metabolomic changes in CLN3 mutant organoids, when compared to controls, which indicate imbalances during brain development. Here, we provide a proof of principle that our cellular model recapitulates key disease features in different cell types in vitro and is thereby suitable for modeling JNCL.

\section{Results}

Generation of a CLN3 mutant isogenic pair

To insert the c.1054C $\rightarrow \mathrm{T}$ pathologic variant on the CLN3 gene, we designed a 21 bp sgRNA that targets the exon 13 of the human CLN3 locus to produce a Cas9- induced double-strand break. In order to visualize and follow the genotypic outcome of the editing, excluding random integration, we applied the FACS assisted CRISPR-Cas9 genome editing (FACE) pipeline $[3,35]$. Briefly, to promote homologous recombination, we created two double-stranded DNA donors containing a positive selection module with either EGFP or dTOMATO and the puromycin resistance gene, flanked by approximately $1 \mathrm{~Kb}$ homology arms. The left homology arm contained, in both donors, the c.1054C $\rightarrow \mathrm{T}$ single nucleotide change for a homozygous outcome (Fig. 1a). In a first step, the constructs were introduced into the genome of healthy control hiPSCs. Puromycin-selected cells were collected, and a double-positive population was selected through several rounds of cell sorting, excluding the ones containing random integration events (BFP positive). Despite the fact that biallelic targeting occurred initially in a frequency of $0.6 \%$, the entire population could be enriched (Fig. 1b). In a second step, the positive selection module was excised by exogenously expressing the excision-only variant of the PiggyBac transposase [47]. Subsequently, the doublenegative population could be purified by cell sorting (Fig. 1c). Precise introduction of the mutation in homozygosis was confirmed by Sanger sequencing. Additionally, silent mutations in the PAM sequence, introduced to shield the site from Cas9 after insertion, were also present and in homozygosis, representing a successful editing procedure (Fig. 1d). Pluripotency of the lines was evaluated by immunostaining for OCT4, SOX2, TRA1-81, TRA1-60 and SSEA4 (Additional file 1: Figure S1b). Karyotype 
a
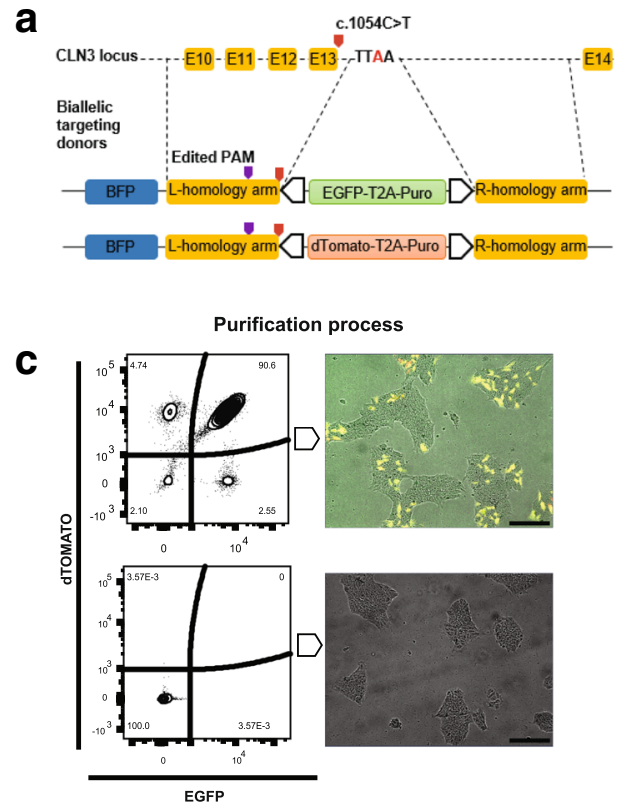

e

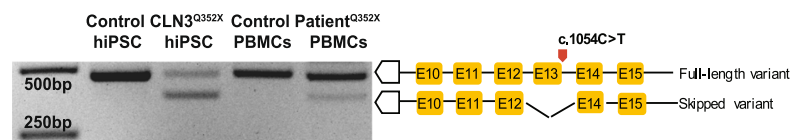

Enrichment process

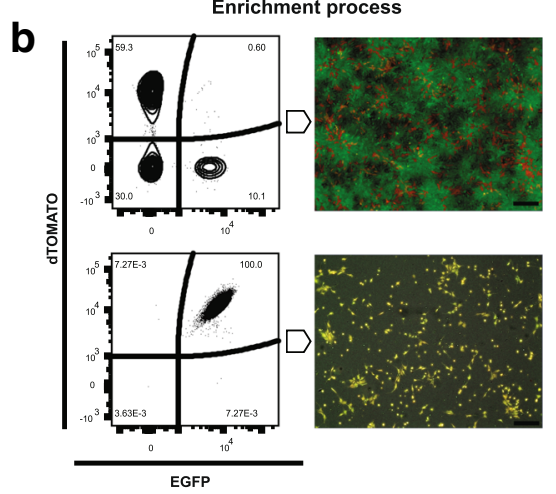

d
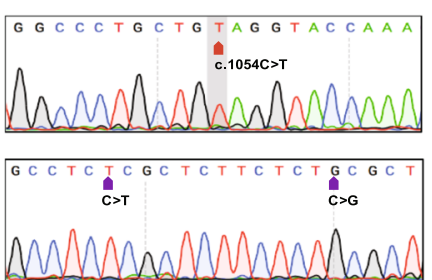

$\mathbf{F}$

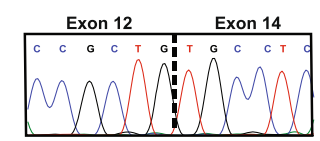

Fig. 1 Gene editing-mediated introduction of the C.1054C > T mutation in the CLN3 locus alters splicing. a Representation of biallelic donors containing distinctive fluorescent positive selection modules (PSM) and the targeted genomic region. $\mathbf{b}$ FACS plots depicting the different populations through the steps of the editing process. The upper panel represents the line after the integration of the PSM and the lower panel, the enrichment of the line after several rounds of sorting for the double-positive population. Plots are accompanied in the right side by microscope images for proper visualization of each step. Scale bars, $200 \mu \mathrm{m}$. c FACS plots depicting the different populations through the steps of the editing process. The upper panel represents the line after the excision of the PSM and the lower panel, the purification of the line after several rounds of sorting for the double-negative population. Plots are accompanied in the right side by microscope images for proper visualization of each step. Scale bars, $200 \mu \mathrm{m}$. d Sanger sequencing chromatogram of the obtained polyclone highlighting the introduction of the mutation and the silent PAM modifications. e Representative RT-PCR gel showing the different band amplification sizes in the CLN3 ${ }^{\text {Q352X }}$ mutant hiPSCs and patient PBMCs, as compared to the controls. $\mathbf{f}$ Second band corresponds to an exon-skipping event of the mutated exon. Sanger sequencing confirmed the junction between neighboring exons

assessment revealed no major abnormalities in both the edited polyclone and the parental control lines (Additional file 1: Figure S1c). Potential off-target loci for the utilized sgRNA (refer to CLN3-QtoO-B in methods section) were predicted by the CRISPR/Cas9 target online predictor, CCTOP [73]. For the top seven off-target sites, we designed a pair of primers to amplify between 200 and $900 \mathrm{bp}$ of the predicted region. Sanger sequencing analysis revealed no detectable off-target modifications induced in the isogenic pair by the genome editing process (Additional file 6: Table S2).

\section{The CLN3 p.Q352X mutation causes a novel splicing variant}

The c.1054C $\rightarrow \mathrm{T}$ mutation is located at the last codon of exon 13 from the CLN3 gene and it produces a premature termination codon (PTC), responsible for generating a truncated protein [55]. Brooks and colleagues reported a frequency of $2.8 \%$ of PTC-inducing mutations for the CLN3 gene [12]. In addition, PTCs are shown to frequently induce alternative splicing, often leading to skipping of the PTCcontaining exon [17]. To test this hypothesis in the here generated isogenic line, reverse-transcription PCR (RT-PCR) analysis using primers that amplified exons 11 to 15 , revealed two different cDNA amplicons in the CLN3 mutant hiPSCs, one with the expected normal size $(480 \mathrm{bp})$ and a product of around $100 \mathrm{bp}$ smaller in size (Fig. 1e). Sanger sequencing confirmed that the smaller product corresponded to an exon-skipped variant, which lacked exon 13 (Fig. 1f). To our knowledge, the potential affection of the splicing by the p.Q352X mutation was not previously described. Therefore, we further investigated this alternative splicing variant in peripheral blood mononuclear cells (PBMCs) extracted from a patient carrying the same mutation in homozygosity. This confirmed the same splicing pattern, although the 
proportion of the two variants seems to differ, which might be caused by cell type specific differences (Fig. 1e).

\section{CLN3 ${ }^{\text {Q352X }}$ hiPSC-derived endothelial cells recapitulate JNCL disease hallmarks}

In juvenile neuronal ceroid lipofuscinosis the ultrastructural visualization of storage material, in a distinctive pattern, called fingerprint profiles (FPPs) constitutes a disease hallmark. Following the study from Pérez-Poyato and colleagues, that used endothelial cells (ECs) to characterize storage inclusions in CLN3 patients [62], we derived endothelial cells (ECs) from the here generated hiPSC isogenic pair and investigated the potency of the $\mathrm{CLN}_{3}{ }^{\mathrm{Q} 352 \mathrm{X}}$ mutant line to recapitulate storage disorder pathology. Cell differentiation towards the endothelial lineage was assessed by immunostaining of the endothelial cell surface marker CD31, showing a similar staining distribution in both cell lines (with a mean fluorescence intensity of $0.76 \pm 0.05$ in the Control and $0.84 \pm 0.04$ in the $\mathrm{CLN}^{\mathrm{Q}}{ }^{\mathrm{Q} 32 \mathrm{X}} \mathrm{ECs}$ ) (Fig. 2a). The different cellular compartments and organelles were examined by transmission electron microscopy (TEM) after 15 days of differentiation. We observed major autophagic structures, constituted by a single limiting membrane and cellular components at various stages of degradation, which represent autophagic vacuoles (AVs) (Fig. 2b). Quantification of the percentage of AVs per cell profile highlighted a significantly increased presence of these structures in the $\mathrm{CLN}^{\mathrm{Q} 352 \mathrm{X}}$ mutant line $(15.57 \pm 2.7 \%$ in the Control and $55.27 \pm 12.9 \%$ in the $\left.\mathrm{CLN}^{\mathrm{Q} 352 \mathrm{X}} \mathrm{ECs}\right)$. Although the AV area normalized to total cell area was not significantly different, there was a trend towards an increased size of the AVs in the case of the mutant cells $(0.64 \pm 0.15 \%$ in the Control versus $5.38 \pm 4.02 \%$ in the $\mathrm{CLN}^{\mathrm{Q} 352 \mathrm{X}}$ ECs) (Fig. 2c). Strikingly, we were also able to find electron dense storage material with fingerprint patterned morphology in the $\mathrm{CLN}_{3}{ }^{\mathrm{Q} 352 \mathrm{X}}$ mutant ECs, which looked very similar to the storage material typically observed in JNCL patients (Fig. 2d) [49, 54]. The observation of the classical storage material by TEM prompted us to further examine the ECs by immunostaining of the subunit $\mathrm{c}$ of the mitochondrial ATP synthase (SCMAS) and the lysosomalassociated membrane protein 1 (LAMP1). We observed a significantly higher amount of LAMP1 and SCMAS positive structures in the $\mathrm{CLN}^{\mathrm{Q} 352 \mathrm{X}}$ mutant ECs, as well as a significantly higher degree of co-localization between the two structures, indicative of an increased presence of SCMAS inside the lysosomes in the case of the mutant ECs, which may be part of the aberrant storage material (Fig. 2e, f). Alterations were not only restricted to the lysosomal compartment. Golgi stacks were often broadened up in the case of the $\mathrm{CLN}^{\mathrm{Q} 352 \mathrm{X}}$ mutant cells (Fig. 2g). Consequently, we immunostained to visualize the cis-Golgi marker GM130 (Fig. 2h). Image analysis revealed a lower proportion of Golgi structures in the $\mathrm{CLN}^{\mathrm{Q} 352 \mathrm{X}}$ mutant ECs but with a higher degree of ramification, as seen by the increased amount of pixels that form the Golgi skeleton and the average of ramification points (or nodes) per Golgi structure (Fig. 2i). Evaluation of other organelles revealed no ultrastructural abnormalities in the endoplasmic reticulum (ER) (Additional file 2: Figure S2a), the endosomal membranes (early endosomes and late endosomes/lysosomes) (Additional file 2: Figure S2b), or the mitochondria (Additional file 2: Figure S2c) in the $\mathrm{CLN}^{\mathrm{Q} 352 \mathrm{X}}$ ECs. However, immunostaining for the Ras-related protein Rab-7a (RAB7) (Additional file 2: Figure S2d), a small GTPase that plays a key role in regulating the transport from early to late endosomal compartments [29], highlighted a significantly decreased amount of RAB7 positive structures, which are also smaller in size on average, in the CLN3 ${ }^{\mathrm{Q} 352 \mathrm{X}}$ ECs compared to the Control cells (Additional file 1: Figure S2e). As RAB7 is a key regulator of the transport into degradative compartments [15], these results are a further indication for defects in the maturation and/or trafficking of the endolysosomal compartments in CLN3 ${ }^{\mathrm{Q} 352 \mathrm{X}}$ ECs.

\section{Severe failure to develop in $\mathrm{CLN}^{\mathrm{Q} 352 \mathrm{X}}$ hiPSC-derived cerebral organoids}

Little is known about early brain development in the context of JNCL. To evaluate whether there is an early developmental component to CLN3 disease, we used cerebral organoids as a state of the art human model to study brain development and disease $[1,45,51,66]$. In this regard, we generated cerebral organoids from the here generated hiPSC isogenic pair [44] and we cultured them up to the stage where cortical markers are typically expressed (55 days of differentiation) [69]. Initial screening involving size evaluation of individual organoids up to the stage of neural induction (day 10) revealed no major differences (Additional file 3: Figures S3a, b). However, upon matrix embedding and initiation of the differentiation (day 11), the average size of CLN3 ${ }^{\mathrm{Q} 352 \mathrm{X}}$ organoids was slightly, but significantly reduced, in comparison to the isogenic control without the mutation (Fig. 3a, b). Interestingly, about half of the CLN3 ${ }^{\mathrm{Q} 352 \mathrm{X}}$ organoids completely failed to grow and develop further until the end-point of the differentiation (Fig. 3c, d). This severe phenotype was observed repeatedly across several organoid generations. The other half of mutant organoids, which escaped this severe defect were used for the analysis of more subtle alterations. In these fullydeveloped cerebral organoids, with and without mutant CLN3, comparable expression of brain identity markers, such as the forebrain marker FOXG1 were detected, either via immunofluorescence or western blot. Neuronal 


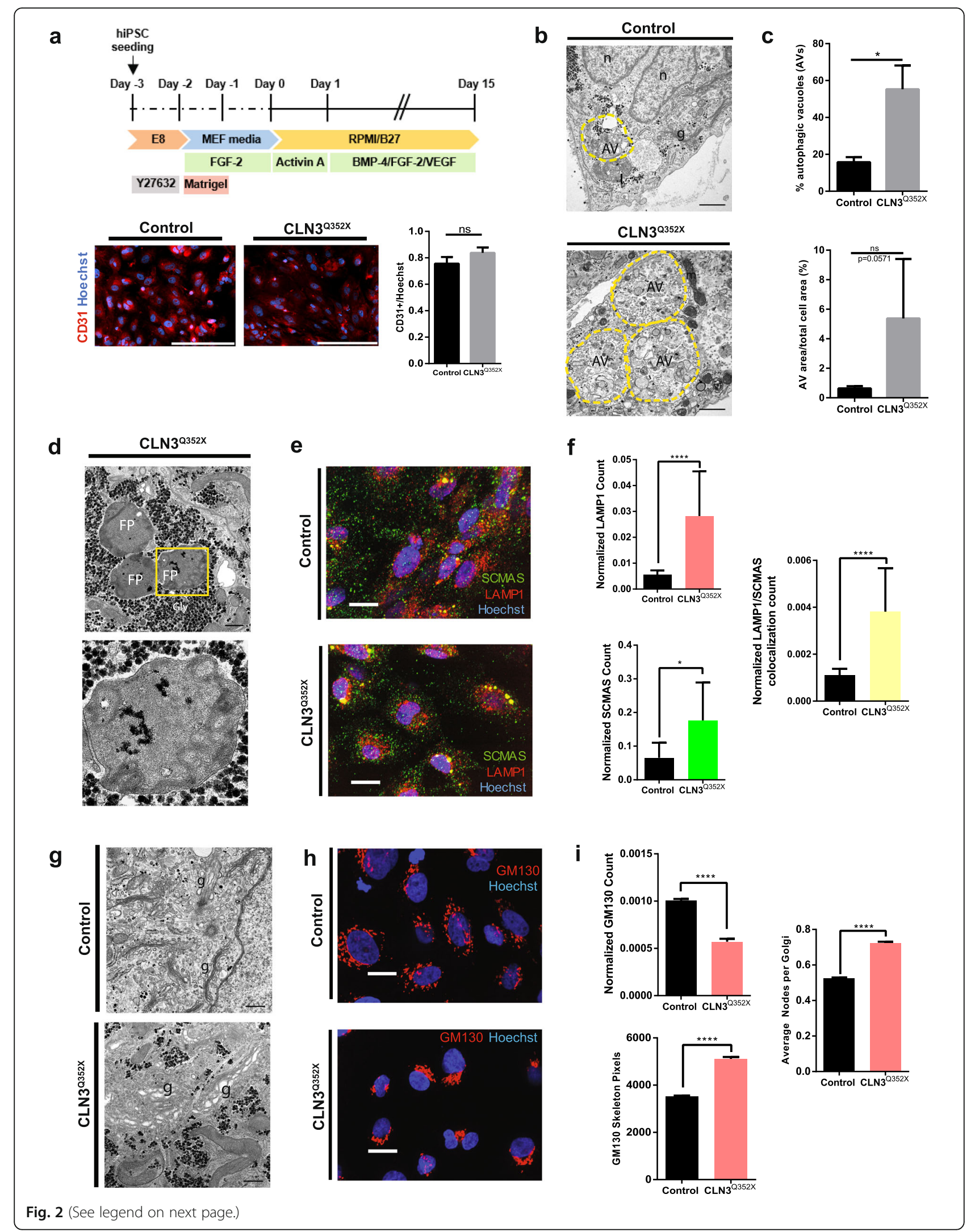


(See figure on previous page.)

Fig. 2 The CLN3 C.1054C > T introduction generated classical JNCL phenotypes in vitro. a Protocol for endothelial cell (EC) derivation from hiPSCs and representative immunofluorescence pictures of CD31 positive endothelial cells for characterization. Scale bar, $200 \mu \mathrm{m}$. Quantification of CD31 positive cells performed in Control and CLN3 ${ }^{\mathrm{Q} 352 \mathrm{X}}$ ECs. Mean fluorescence intensity of the CD31 channel normalized to the nuclear (Hoechst positive) signal was calculated for 12 random fields per condition using ImageJ (NIH) (Welch's test; ns, not significant). Data is shown as mean \pm SEM. b Representative TEM pictures highlighting increased presence of autophagic vacuoles (AVs, yellow dashed line) in the CLN3 ${ }^{\mathrm{Q} 352 \mathrm{X}}$ ECs. Other organelles (n, nuclei; g, Golgi; I, lysosome; m, mitochondria). Scale bars, $1 \mu \mathrm{m}$. b Quantification of the percentage of AVs evaluated as the number of events in 56 Control and $52 \mathrm{CLN3}^{\mathrm{Q} 352 \mathrm{X}}$ random cell profiles. Area of the AVs was calculated on ImageJ (NIH) by manually delineating the perimeter as regions of interest (ROI). AV areas were normalized to the total area of the cell containing them. All measurements were corrected for the magnification of the pictures if needed. Comparison between genotypes was performed by a Mann-Whitney test $\left({ }^{*} p<0.05 ; n s ; n o t\right.$ significant). Data is shown as mean \pm SEM. $\mathbf{d}$ Representative TEM pictures of fingerprint inclusions (FPPs) seen in CLN3 ${ }^{\text {Q352X }}$ ECS (Gly, glycogen deposits). Scale bar, $500 \mathrm{~nm}$. Zoomed in picture (yellow dashed square) for better visualization of membrane stacks. Scale bar, $200 \mathrm{~nm}$. e Highcontent imaging of SCMAS (green), LAMP1 (red) structures, and co-localization events (yellow) in Control and CLN3 ${ }^{\mathrm{Q} 352 \mathrm{X}}$ ECs. Scale bar, $200 \mu \mathrm{mm}$. f SCMAS, LAMP1 and colocalization counts normalized to the nuclear (Hoechst positive) area of each field. Differences were evaluated using MannWhitney test $\left(^{*} p<0.05,{ }^{* * * *} p<0.0001\right)$. Data is represented as mean \pm SEM of three technical replicates. $\mathbf{g}$ Representative TEM images of structural differences in the Golgi compartment in the Control (stacked) and the CLN3 ${ }^{\mathrm{Q} 352 \mathrm{X}}$ (dilated) ECs (g, Golgi; m, mitochondria; gly, glycogen). Scale bars, $500 \mathrm{~nm}$. h High-content imaging of Golgi (GM130 positive) structures (red). Scale bar, $200 \mu \mathrm{m}$. i Quantification of GM130 counts, normalized to the nuclear (Hoechst positive) area of each field. GM130 positive structures present an increased ramification in terms of skeleton pixels and average nodes per Golgi structure. Comparison performed with Mann-Whitney test (**** $p 0.0001$ ). Data is represented as mean \pm SEM of three technical replicates

marker MAP 2 was also present and neuronal identity corresponding to several cortical layers, like SATB2 expression for later-born superficial-layer identity neurons, and early-born deep-layer identity marked by the expression of CTIP2 and TBR1 were detectable at comparable levels. Moreover, they also expressed neural stem cell markers SOX2 and Nestin (Fig. 3e, f) [68]. However, mRNA levels of FOXG1, SATB2 and TBR1 genes were found significantly downregulated in the $\mathrm{CLN}_{3}{ }^{\mathrm{Q} 352 \mathrm{X}}$ mutant phenotype (Additional file 5: Figure S5a). Finally, TEM analysis highlighted the presence of nerve fibers with neurofilaments and synaptic vesicles in both genotypes (Fig. 3g). Based on these results we conclude that about half of the $\mathrm{CLN}_{3}{ }^{\mathrm{Q} 352 \mathrm{X}}$ organoids were able to escape severe development defects which prohibit their development into cerebral organoids. We decided to further analyse these escapers for more subtle defects that might be caused by presence of the $\mathrm{CLN} 3^{\mathrm{Q} 352 \mathrm{X}}$ mutation.

\section{CLN3 mutant cerebral organoids present lysosomal alterations, storage pathology and astrocytosis} Ultrastructural analysis of $\mathrm{CLN}^{\mathrm{Q} 352 \mathrm{X}}$ mutant cerebral organoids confirmed the presence of pathological storage material, as seen by the increased presence of AVs (Fig. 4a) of significantly greater area in the case of the CLN3 ${ }^{\mathrm{Q} 352 \mathrm{X}}$ organoids $(4.9 \pm 0.75)$ compared to the Control organoids $(3.28 \pm 0.86)$ (Fig. 4b). Strikingly, we were also able to find intracytoplasmic and electron dense storage material with fingerprint morphology in the $\mathrm{CLN} 3^{\mathrm{Q} 352 \mathrm{X}}$ organoids mutant organoids [54], as well as structures resembling curvilinear bodies (CVB) [9] (Fig. 4c, d). Pathological intracellular deposits in JNCL are typically autofluorescent [70] and composed mainly of subunit $\mathrm{c}$ of the mitochondrial ATPase (SCMAS) [60]. However, autofluorescence analysis in organoid sections with confocal laser excitation at $568 \mathrm{~nm}$ [52] did not reveal any increase in the $\mathrm{CLN}^{\mathrm{Q} 352 \mathrm{X}}$ mutant organoids (Additional file 3: Figures S3c, d). SCMAS protein levels were also not significantly increased (Additional file 3: Figures S3e, f). To determine whether the storage material results in an impairment of lysosomal functionality, we analyzed the protein levels of TPP1/CLN2, a serine protease in the lysosome, which interacts with CLN3 [77], and found a significant increase in $\mathrm{CLN}_{3}{ }^{\mathrm{Q} 352 \mathrm{X}}$ organoids (Fig. 4e, f). However, at the gene expression level, mRNA counts for the TPP1 gene were significantly decreased in the $\mathrm{CLN}^{\mathrm{Q} 352 \mathrm{X}}$ mutant organoids (Additional file 5: Figure S5b). Additionally, CLN3 has also been implicated in the regulation of other proteases in the lysosome, including Cathepsin D (CTSD/CLN10) $[16,26]$. We detected a significantly decreased concentration of CTSD in lysates from $\mathrm{CLN}{ }^{\mathrm{Q} 352 \mathrm{X}}$ cerebral organoids (Fig. 4g), suggesting that functional alterations at the lysosomal level are already present at this time-point in this developmental model. This is important because not only these proteins are thought to interact with the CLN3 gene, but mutations in TPP1, as well as CTSD cause late-infantile and congenital NCL, respectively [6]. Additionally, we found increased astrocytosis, indicated by the significant increase of GFAP+ cells, in CLN3 $3^{\mathrm{Q} 352 \mathrm{X}}$ mutant organoids (Fig. 4h, i), which has been often reported in CLN3 deficient mice brains $[8,61,63]$. Differences in MAP 2-positive neuronal areas were not significant, however, a trend towards a decreased neuronal areas that may be due to an increased astrocytosis could be observed (Fig. 4i). Furthermore, we were not able to detect increased percentages of apoptotic TUNEL+ DAPI+ cells in CLN3 ${ }^{\mathrm{Q} 352 \mathrm{X}}$ organoids (Additional file 3: Figures $\mathrm{S} 3 \mathrm{~g}$ and $\mathrm{h}$ ), and programmed necrosis (or necroptosis) marker levels, such as kinases receptorinteracting proteins 1 and 3 (RIP1 and RIP3) [7], were not significantly different from the ones in the Control organoids 
a
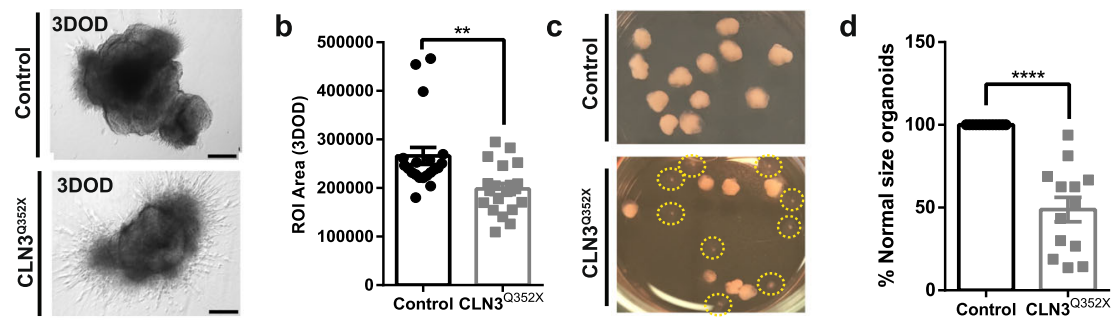

e

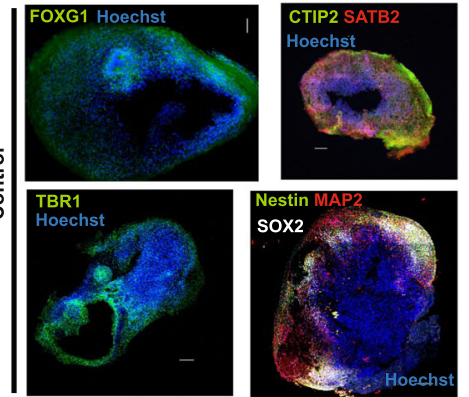

f
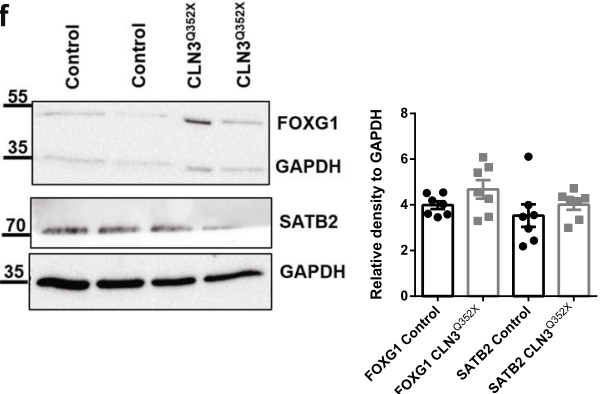
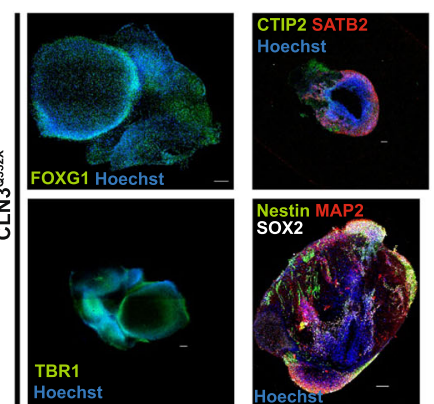

g

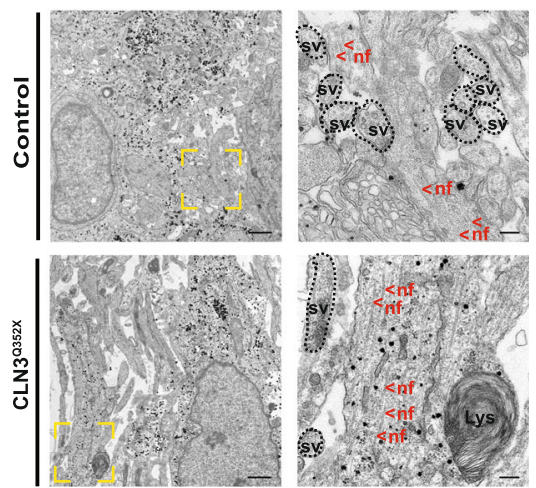

Fig. 3 A large fraction of CLN3 ${ }^{Q 352 X}$ hiPSC-derived cerebral organoids exhibit impaired growth. a CLN3 ${ }^{\mathrm{Q} 352 \mathrm{X}}$ organoids fail to expand and form neuroectodermal protrusions at three days of differentiation (3DOD) compared to the Control organoids. Scale bar, $200 \mu \mathrm{m}$. b Size measurements of organoids at 3DOD show a decrease in the area of the CLN3 ${ }^{\text {Q352X }}$ organoids. Area was calculated on ImageJ (NIH) by manually delineating the perimeter as regions of interest (ROI). Data points represent single organoids from three independent derivations (total $n=20$ organoids). Comparison was performed applying a Mann-Whitney test between the two groups. c A proportion of CLN3 ${ }^{\mathrm{Q} 352 \mathrm{X}}$ organoids remains underdeveloped after 55DOD. Small Matrigel-embeded organoids are highlighted with a dashed yellow circle. d Quantification of the percentage of $C L N 3^{\mathrm{Q} 352 \mathrm{X}}$ mutant organoids reaching a complete development. Data points represent percentage per organoid derivation (total $n=13$ different organoid generations). Comparison between the two groups was performed with an unpaired t-test with Welch's correction. For B and $\mathrm{D}$, data is represented as mean $\pm \operatorname{SEM}\left(p<0.01^{* *}, p<0.0001^{* * * *}\right)$. e Fully developed organoids from both Control and CLN3 ${ }^{\mathrm{Q} 352 \mathrm{X}}$ mutant genotypes express forebrain marker FOXG1 (green) and cortical layer identities: SATB2 (red) for later-born superficial-layer identity and CTIP2 and TBR1 for early-born deep-layer identity (CTIP2 and TBR1, both green), at 55DOD. They also express the neuronal marker MAP 2 (red) and neural stem cell markers SOX2 (white) and Nestin (green). Scale bars, $200 \mu \mathrm{m}$. f. Representative western blots for the some of the markers mentioned above. Quantifications show similar expression in fully developed CLN3 ${ }^{\mathrm{Q} 352 \mathrm{X}}$ organoids compared to the Controls at 55DOD. Data points represent single different organoids ( $n=7$ different organoids per condition). $\mathbf{g}$ Representative TEM pictures of the ultrastructural neuronal features of both organoid cultures at the same stage. Nerve fibers are highlighted in a yellow dashed boxed and higher magnification picture is offered at the right side to visualize neurofilaments ( $n f ;<$, both in red), which are present in both genotypes, as well as synaptic vesicles (sv; black dashed circles). Scale bars represent $1 \mu \mathrm{m}, 200 \mathrm{~nm}$ for magnified areas

(Additional file 3: Figures S3i, j). In summary, these data reveal that we can detect disease relevant early alterations, particularly concerning lysosomal function in $\mathrm{CLN}^{\mathrm{Q} 352 \mathrm{X}}$ mutant cerebral organoids, which may precede more severe phenotypes including cell death.

\section{Whole-transcriptome analysis reveals impaired} development in $\mathrm{CLN}^{\mathrm{Q} 352 \mathrm{X}}$ cerebral organoids

Comparative transcriptomic analyses in the context of CLN3 disease are very limited [32]. Therefore, we used those CLN3 mutant organoids that were able to develop 
a

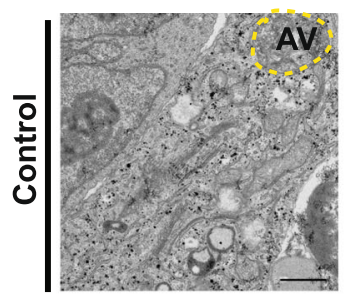

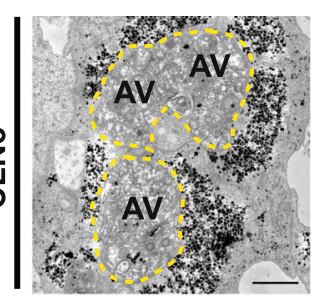

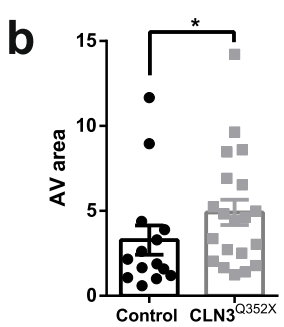

C

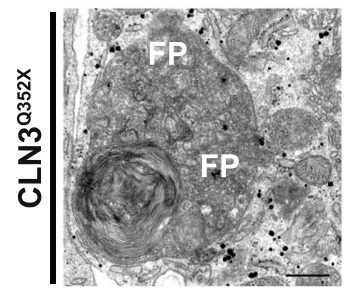

d

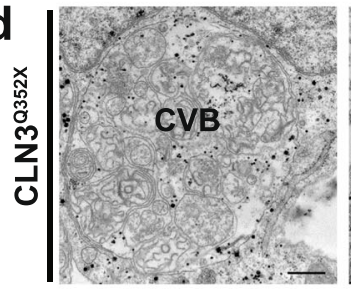

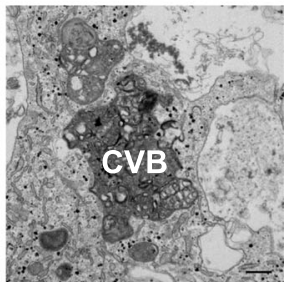
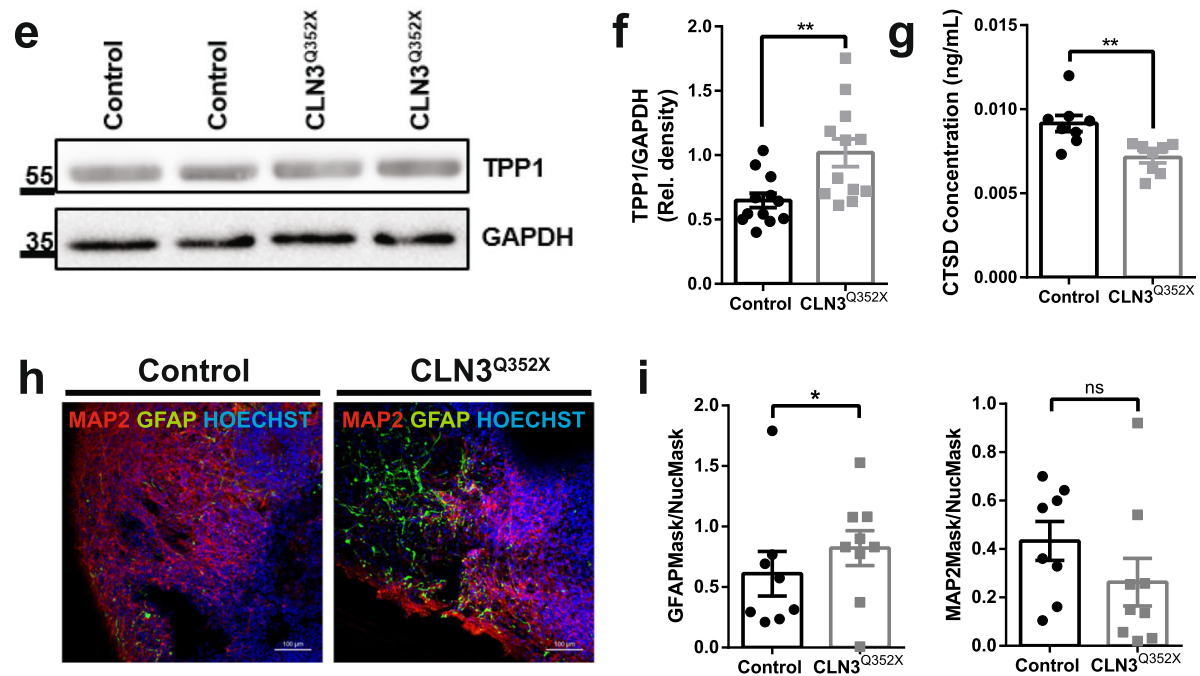

Fig. 4 JNCL cerebral organoids recapitulate disease hallmarks in the neuronal tissue. a Representative TEM pictures highlighting increased presence of autophagic vacuoles (AVs, dashed yellow line) in the CLN3 ${ }^{\mathrm{Q} 352 \mathrm{X}}$ organoids. Scale bars, $1 \mu \mathrm{m}$. b Quantification of AV area, measured on ImageJ by region of interest (ROI) definition, reveals a significant increase in the size of the vacuoles in the CLN3 ${ }^{\mathrm{Q} 352 \mathrm{X}}$ organoids. Comparison from 14 Control and $20 \mathrm{CLN} 3^{\mathrm{Q} 352 \mathrm{X}}$ random AV profiles was done using Mann-Whitney test, ${ }^{*} p<0.05$. Data points indicate single AVs measured per condition. c Representative TEM pictures of fingerprint inclusions (FPPs) detected exclusively in the CLN3 ${ }^{\text {Q352X }}$ organoids. Scale bar, $500 \mathrm{~nm}$. $\mathbf{d}$ Representative EM pictures of curvilinear bodies (CVB) found exclusively in the CLN3 ${ }^{\text {Q352X }}$ organoids. Scale bar, $500 \mathrm{~nm}$. e Representative western blot staining for TPP1 showing a slight increase in the CLN3 ${ }^{\mathrm{Q} 352 \mathrm{X}}$ organoids. $\mathbf{f}$ Quantification of the TPP1 levels from western blots. Data points represent individual organoids ( $n=12$ organoids from at least three independent derivations) (Welch's test; ${ }^{* *} p<0.01$ ). $\mathbf{g}$ ELISA quantification of CTSD protein levels. Data points represent single different organoids ( $n=8$ organoids generated in at least three independent derivations) (Welch's test; ${ }^{* *} p<0.01$ ). $\mathbf{h}$ Astrocytosis shown by increased GFAP positive (green) staining in the CLN3 ${ }^{\mathrm{Q} 352 \mathrm{X}}$ organoids compared to the controls. Scale bars, $100 \mu \mathrm{m}$. i Image analysis of GFAP and MAP 2 positive staining in organoid sections, normalized by nuclear (Hoechst positive) staining. Data points represent different organoid sections ( $n=8$ Control and 9 CLN3 ${ }^{\mathrm{Q} 352 \mathrm{X}}$ sections taken from different organoids generated in at least three independent derivations). Comparison evaluated by Kolmogorov-Smirnov test ( ${ }^{*} p<0.05$; ns, not significant). Data in $B, F, G$ and I is shown as mean \pm SEM

and performed whole-transcriptome RNA-seq analysis to detect early disease signatures at the gene expression level. In order to gain a deeper understanding of JNCL disease-related dysregulations at a systems-level, we employed a differential gene regulatory network (GRN) based analysis to reconstruct phenotype-specific networks representing the $\mathrm{CLN} 3^{\mathrm{Q} 352 \mathrm{X}}$-diseased (mutant) and Control (healthy) phenotypes [5, 14, 65]. Differential expression analysis (DEA) resulted in 972 genes to be significantly (Benjamini Hochberg corrected $p$-value $\leq 0.05$ and $\log \mathrm{FC}>1$ ) differentially expressed (up- and down-regulated) between the Control and the $\mathrm{CLN}^{\mathrm{Q} 352 \mathrm{X}}$ mutant cerebral organoids (Fig. 5a). The hierarchical clustering analysis revealed the presence of 

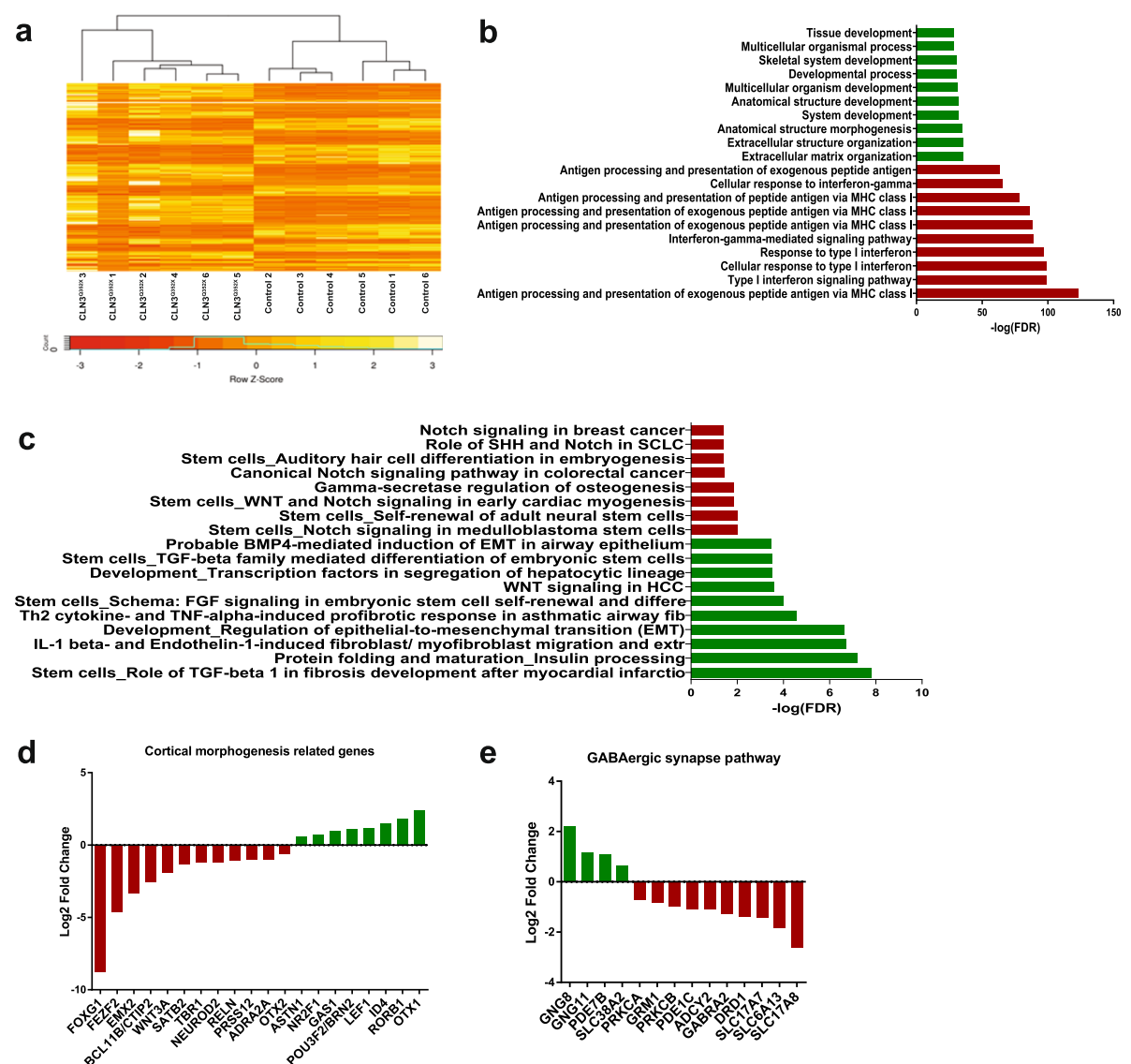

Fig. 5 Whole-transcriptome analysis reveals impaired development in $\mathrm{CLN} 3^{\mathrm{Q} 352 \mathrm{X}}$ cerebral organoids. a Heatmap clustering the differentially expressed genes between Control (healthy) and CLN3 ${ }^{\mathrm{Q} 352 \mathrm{X}}$ (mutant) brain organoids. b Gene enrichment analysis of CLN3 ${ }^{\mathrm{Q} 352 \mathrm{X}}$ network. Genes that are upregulated in disease phenotype were significantly enriched in cellular processes highlighted in green, while downregulations are depicted in red. c Pathway enrichment analysis of the CLN3 ${ }^{\mathrm{Q} 352 \mathrm{X}}$ network. Pathway up-regulations are highlighted in green, while downregulations are marked in red. $\mathbf{d}$ Log 2 Fold change expression values for genes related to brain development and cortical morphogenesis were generally decreased in CLN3 ${ }^{\mathrm{Q} 352 \mathrm{X}}$. e Log 2 Fold change expression values for genes related to synapses, which were mostly downregulated in the $\mathrm{CLN3}{ }^{\mathrm{Q} 352 \mathrm{X}}$ organoids. Genes in D and $\mathrm{E}$ are specifically differentially in our dataset (but not necessarily present in the networks) and belong to the pathways extracted from Pathway unification database (PathCards)

two clearly distinct groups, with different gene expression signatures, which correlate with the presence of the CLN3 $3^{\mathrm{Q} 352 \mathrm{X}}$ mutation (Fig. 5a). The reconstructed CLN3 ${ }^{\mathrm{Q} 352 \mathrm{X}}$-diseased network comprised 353 genes and 641 interactions, whereas the Control healthy network contained 298 genes and 399 interactions (Additional file 4: Figures S4a, b). Interestingly, gene ontology (GO) analysis of the $\mathrm{CLN}_{3}{ }^{\mathrm{Q} 352 \mathrm{X}}$-diseased network revealed that most of the up-regulated genes in the network were significantly enriched in cellular processes related to development, such as tissue development (GO: 0009888, FDR:5.84E-29), multicellular organism development (GO:0007275, FDR:6.67E-32) and extracellular matrix (ECM) organization (GO:0030198, FDR:3.68E36 ). On the other hand, most downregulations were targeting biological processes related to antigen processing and presentation via the class I major histocompatibility complex (MHC I) (GO:0002480), FDR: 9.73E-124)
(Fig. 5b). Interestingly, antigen presenting cell abnormalities have been previously reported in a CLN3-deficient mouse model [31]. Furthermore, pathway enrichment analysis highlighted significant dysregulations in molecular pathways related to stem cells and development (Fig. 5c). Particularly, the TGF-beta, WNT and BMP signaling pathways were found to be significantly associated with the disease specific network, these signaling pathways are well characterized for their fundamental roles in embryonic development and homeostasis [72, 80, 82]. This suggested that dysregulations of developmental pathways and processes in $\mathrm{CLN}^{\mathrm{Q} 352 \mathrm{X}}$ cerebral organoids constitute a disease signature, already at early stages during the brain development. Additionally, we analyzed the expression levels of genes related to cortical morphogenesis and found that transcription factors critical in central nervous system development, such as FOXG1 [30], FEFZ2 [22], CTIP2 [56], SATB2 [11], 
TBR1 [21] or NEUROD2 [57] were predominantly downregulated in the $\mathrm{CLN}^{\mathrm{Q} 352 \mathrm{X}}$ organoids (Fig. 5d). Moreover, expression of genes encoding relevant synaptic proteins, such as amino acid transporters from the SLC6 and the SLC17 families, as well as neurotransmitter receptors, such as $\gamma$-Aminobutyric acid (GABA) receptor GABRA2 and dopamine receptor DRD1, were also decreased (Fig. 5e). These downregulations are indicative of specific alterations in cortical neuronal specification and synapse formation in $\mathrm{CLN}_{3}{ }^{\mathrm{Q} 32 \mathrm{X}}$ organoids.

\section{$\mathrm{CLN3}^{\mathrm{Q} 352 \mathrm{X}}$ cerebral organoids display early synaptic and neurotransmitter defects}

Previous studies in mouse models reported changes in presynaptic release and region-dependent neural network activity dysfunction due to CLN3 deficiency, especially affecting GABAergic and glutamatergic transmission, particularly in the amygdala, hippocampus, and cerebellar networks [13, 28]. Immunofluorescence stainings for synaptophysin (presynaptic marker) and postsynaptic density protein 95 (PSD95; postsynaptic marker) revealed a significant decrease in presynaptic and postsynaptic counts in $\mathrm{CLN}^{\mathrm{Q} 352 \mathrm{X}}$ mutant organoids (Fig. 6a, b). These synaptic marker proteins have been shown to manifest changing patterns during the human frontal cortex development [24], but alterations in the levels of these proteins in the cerebral organoids model complement the gene expression data (Fig. 5) highlighting impairments in synapse formation. To gain further insights, we compared the metabolic profiles of $\mathrm{CLN}^{\mathrm{Q} 352 \mathrm{X}}$ mutant cerebral organoids to the isogenic controls. Unsupervised clustering separated both genotypes, highlighting dysregulations in 66 different metabolites between the two, with most of the metabolites being less represented in the $\mathrm{CLN}_{3}{ }^{\mathrm{Q} 352 \mathrm{X}}$ mutant organoids (Fig. 6c). From these metabolites, a total of 31 were identifiable (Additional file 6: Table S3). Within these metabolites, we detected a significant decrease in some amino acids, such as tryptophan and lysine and interestingly also in neurotransmitters, like the $\gamma$-aminobutyric acid (GABA). Creatinine, a putative metabolite biomarker for several neurodegenerative diseases [40], was also significantly downregulated in the mutant organoids (Fig. 6d, e, Additional file 6: Table S4). To further demonstrate the downregulation of the GABA system, the vesicular GABA transporter vGAT was checked via immunostaining, revealing a significant decrease in the evaluated $\mathrm{CLN}_{3}{ }^{\mathrm{Q} 352 \mathrm{X}}$ mutant organoid sections (Fig. 6f, g). In summary, these findings reveal an impairment in synapse formation and neurotransmitter production in CLN3 mutant cerebral organoids.

\section{Discussion}

The impact of CLN3 mutations on neurodevelopment is clearly understudied and especially studies using human in vitro models are missing. The advent of iPS cell technologies allows the development of such models, which are especially relevant in rare disease studies, where the paucity of available patient samples limits research development. In this context, the generation of isogenic cell lines by means of CRISPR/Cas9-mediated genome editing is of great importance, especially to isolate the effect of the disease-causing mutations from the patient-specific genetic background. Moreover, advances in three-dimensional organoid cultures foster the use of the human iPS cells to study neurodevelopment $[1,45]$. Here, we generated a CLN3 mutant hiPS cell line by introducing the $\mathrm{c} \cdot 1054 \mathrm{C} \rightarrow \mathrm{T}$ pathologic variant in the CLN3 gene and used organoid technology to obtain a neurodevelopmental in vitro model of CLN3 disease, which recapitulates key disease features and allows to study the impact of the mutation on brain development.

While characterizing the isogenic cell pair, we discovered that the introduced mutation promotes the exclusion of the constitutive mutated exon from the transcript. More importantly, we were able to confirm the existence of the alternative spliced variant in patient cells carrying the same mutation. This phenomenon was not previously reported for this mutation and is most likely occurring in other nonsense CLN3 mutations located in exon boundaries, gaining specifically relevance when designing effective therapeutic strategies. In addition, the value of the here described isogenic pair was further demonstrated by showing distinct diseasespecific phenotypes in different in vitro cultures. Firstly, in this study, hiPSC-derived endothelial cells recapitulate organellar pathology and present JNCL-type storage material. Secondly, hiPSC-derived cerebral organoids also displayed the disease hallmark, finger print material accumulation, interestingly found in combination with curvilinear bodies. Additionally, we show that storage pathology is co-occurring with alterations in the levels of lysosomal enzymes, such as TPP1 and CTSD, which have been described in various pathological conditions such as neurodegenerative lysosomal storage disorders, inflammation, cancer and aging [25, 39]. Taken together, these results validate the here described isogenic pair as a suitable human model for JNCL, which recapitulates key features of disease pathology both in twodimensional non-neuronal and three-dimensional neural cell cultures.

Previous studies using cerebral organoids as model for rare diseases with known genetic risk factors, such as primary microcephaly [45] or Sandhoff disease [1] reported a markedly reduced or increased organoid size, respectively, with impaired neuronal differentiation in patient organoids. We observed that the CLN3 mutant cells were considerably sensitive to the cerebral organoid differentiation, failing to form neuroepithelial buds after the start of differentiation and impairing their complete 


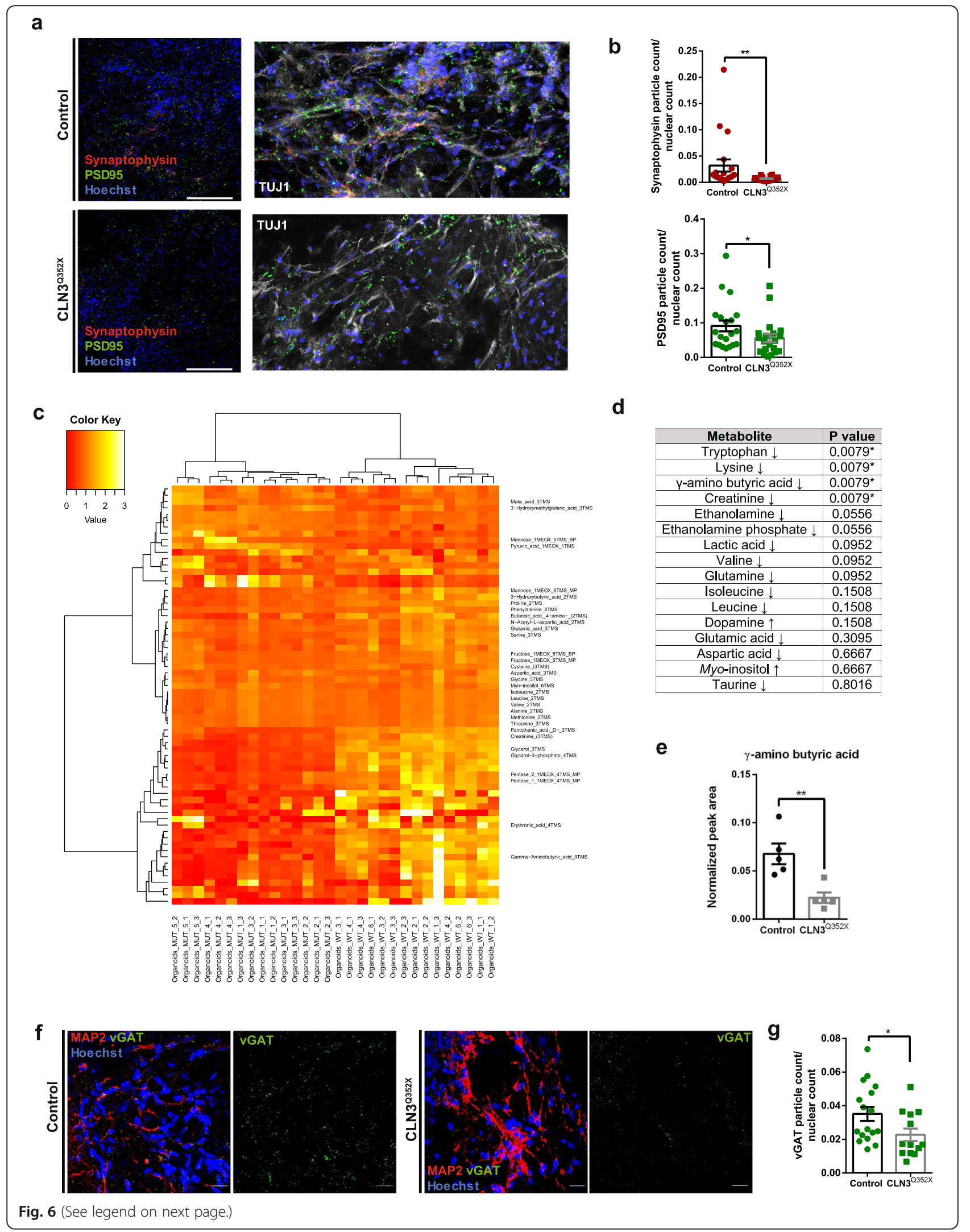




\begin{abstract}
(See figure on previous page.)
Fig. 6 Dysregulations at the synapses might precede other phenotypes in CLN3 ${ }^{\mathrm{Q} 352 \mathrm{X}}$ cerebral organoids. a Representative confocal images of synaptic protein staining for Synaptophysin (red) and PSD95 (green). Scale bars, $200 \mu \mathrm{m}$. Zoomed regions with TUJ1 (white) neuronal staining. b Quantification of the Synaptophysin and PSD95 positive particles normalized to nuclear (Hoechst positive) count. Two regions of interest (ROI) per organoid section were imaged. Following automatic thresholding, the particle analyzer tool from ImageJ (NIH) was used to quantify punctate stainings -PSD95 and Synaptophysin- and the ITCN nuclear count tool to quantify Hoechst positive nuclei. Each data point represents a region of interest (ROI). Total $\mathrm{n}$ per group equals 10 sections taken from organoids generated in at least three independent derivations. Comparison between groups was performed with a Mann-Whitney test $\left(^{* *} p<0.01\right.$; ns, not significant). c Heatmap representing hierarchical clustering of deregulated metabolites between the Control and the CLN3 ${ }^{\mathrm{Q} 352 \mathrm{X}}$ mutant organoids. 5 different pools of 3 organoids were analyzed and 3 technical replicates per measurement were performed. $\mathbf{d}$ Table containing cerebral tissue metabolites. Arrows indicate relative increase or decrease in the $\mathrm{CLN} 3^{\mathrm{Q} 32 \mathrm{X}}$ mutant organoids compared to the controls. Asterisks mark significantly deregulated metabolites in the mutant organoids, corresponding to $p$ values. e Neurotransmitter GABA is particularly downregulated in the CLN3 ${ }^{\text {Q352X }}$ mutant organoids. Significant differences were evaluated with a Mann-Whitney test comparison $\left({ }^{* *} p<0.01\right)$. Data points represent the average of technical replicates per organoid pool. Data in B and E is shown as mean \pm SEM. $\mathbf{f}$ Representative confocal images of inhibitory GABAergic neurons pre-synaptic protein VGAT staining (green) and MAP 2 positive neuronal areas (red). Single VGAT channel is displayed inside a square dashed box for better visualization. Scale bars, $20 \mu \mathrm{m}$. $\mathbf{g}$ Quantification of the vGAT positive particles normalized to Hoechst positive nuclei. Several regions of interest (ROI) per organoid section were imaged. Following automatic thresholding, the particle analyzer tool from ImageJ (NIH) was used to quantify VGAT punctate staining and the ITCN nuclear count tool was used to quantify Hoechst positive nuclei. Each data point represents a region of interest (ROI). Total $n$ per group is 4 sections, one per organoid and each belonging to independent derivations. Comparison between groups was performed with an unpaired t test with Welch's correction $\left({ }^{*} p<0.05\right)$
\end{abstract}

development. It is noteworthy that about half of the CLN3 ${ }^{\mathrm{Q} 352 \mathrm{X}}$ organoids across the different organoid generations presented extremely severe development abnormalities, whereas the rest developed normally in terms of brain and cortical identity. Provided that the starting population for the organoid generation was a pool of gene edited hiPS cells, the maturation failure could be possibly due to different expression levels of the two mutant variants of the CLN3 gene and dissimilar amounts of truncated protein. We hypothesize that compensatory mechanisms present within some of the edited cells may favour their further maturation. Therefore, we used the fully-developed organoids to unravel less severe disease signatures, caused by the presence of the $\mathrm{CLN}^{\mathrm{Q} 352 \mathrm{X}}$ mutation, which may be present already during brain development and precede end-stage lysosomal storage defects. Transcriptomic profiling indicated that pathways involved in cell fate differentiation, proliferation and tissue and system developmental processes were altered in the $\mathrm{CLN}^{\mathrm{Q} 352 \mathrm{X}}$ organoids compared to the controls. A closer look into subsets of genes appointed to specific downregulations affecting pathways related to corticogenesis and synapses. Although fullydeveloped organoids for both genotypes presented similar levels of cortical proteins, such as FOXG1 and SATB2, mRNA levels evaluated at the same time-point revealed downregulations for the corresponding genes in the $\mathrm{CLN}_{3}{ }^{\mathrm{Q} 352 \mathrm{X}}$ mutant organoids. We consider that, at the stage of analysis, the system is already showing alterations at the gene expression level, but is still able to compensate for these alterations by using the available transcripts to produce sufficient levels of the corresponding proteins. On the other hand, recent studies reported alterations in the synaptic compartment in CLN3 animal models revealing neurotransmission defects [28] and molecular regulators of synaptic stability [48]. Changes in proteins involved with synaptic function/stability were previously reported in mouse models of different NCL forms [37]. In the CLN3 mutant organoids, evaluation of pre- and post-synaptic proteins revealed a marked decrease in these proteins, suggesting potential alterations in synapse formation during brain development. To evaluate if synapse defects have an influence on neurotransmitter levels, we studied the metabolome of the mutant cerebral organoids, with special focus on metabolites that are implicated in neurotransmission. We were able to find imbalances particularly related to the GABA metabolism, but also in other relevant cerebral metabolites. This constitutes an interesting finding, since GABAergic interneurons are affected in JNCL disease pathogenesis in various animal models and human patients $[50,53]$. The alterations discovered using the cerebral organoid model support the concept of a developmental component to JNCL pathology. We suggest that dysregulated gene expression in key pathways for development and synaptic alterations, particularly affecting GABAergic populations might precede the accumulation of pathologic storage material and the impairment of the lysosomal enzymatic profile, affecting the trajectory of cortical development in children with JNCL.

The advantage of using gene regulatory networks to link genes based on their interaction at the transcriptional level is that it allows detecting hub genes that could be candidate genes for modulation to revert the disease phenotype and bring the mutant network closer to the healthy one. As a proof of principle, we performed in silico network perturbations [84] to identify the most influential genes in the diseased network [36]. Single and multi-gene perturbations revealed several transcription factors, listed in Additional file 6: Table S5, that play an 
important role in maintaining the diseased phenotype network. Although the predicted genes are not necessarily responsible for disease onset and progression, they are predicted to be able to revert most of the diseased gene expression program upon perturbation and thus would be interesting candidates in future studies that could lead to a significant reversion of the pathologic phenotype upon modulation.

The acquisition of an isogenic pair in the context of a rare disease, such as JNCL, overcomes the primary limitation of access to patient material. Moreover, having hiPSC lines that can be differentiated into several cell types allows the study of the pathogeny and the mechanisms of the disease in the different tissues that may be affected. More importantly, the thorough analysis of a particular mutation tailors the study in a personalized manner, and raises the possibility of discovering specific drugs which can have a direct translational impact in patients carrying this particular mutation [10]. Additionally, the established three-dimensional cerebral organoid model for JNCL fosters human in vitro studies of early developmental consequences of this lysosomal storage disorder, where subtle abnormalities could be found preceding terminal cell death and neurodegeneration.

\section{Materials and methods}

\section{Human iPSC culture}

The characterized Gibco (Cat no. A13777) episomal human induced pluripotent stem cell line was chosen as control line to conduct the genome editing. Cells were normally cultured in Matrigel (Crorning, cat no. 354277) coated plates using Essential 8 medium (Thermo Fisher, cat no. A1517001) with 1\% Penicillin/Streptomycin (Invitrogen, cat no. 15140122). Cell splitting procedures were performed using Accutase (Sigma, cat no. A6964) and plated in the same culturing media, but containing ROCK inhibitor Y-27632 (Merck Milipore, cat no. $688000)$ at $10 \mathrm{uM}$ for $24 \mathrm{~h}$ after dissociation.

\section{Construction of sgRNA vectors and donor plasmids}

Introduction of a disease-causing point mutation into the healthy background A13777 hiPS cell line was performed as previously described [3, 35]. Briefly, sgRNAs targeting sequences to exon 13 of the CLN3 gene were selected in silico using a previously published tool [20]. The selected Cas9 sequences with predicted high activity were then cloned into the pX330 vector (Addgene, 42, 230) as described in Ran et al., 2013 [67]. Bi-allelic targeting of the CLN3 locus was achieved by assembling, using Gibson assembly (NEB, cat no. E2611L), the corresponding homology arms into two different donor scaffolds, carrying either EGFP (Addgene 100,603) or dTomato (Addgene 100,604) fluorescent reporters [3, 35]. Both donors contained the desired modification to achieve a homozygous insertion. The donor vectors were named A235-CLN3-donor-green-Q352X and A236CLN3-donor-red-Q352X. All primers used are indicated in Additional file 6: Table S1 and sgRNA sequence resulting in successful editing was corresponding to CLN3-QtoO-B.

\section{Electroporation of parental cells and selection}

Following normal dissociation, hiPSC were electroporated using the 4D-Nucleofector System (Lonza) and the P3 Primary Cell 4D-Nucleofector ${ }^{\circ} \mathrm{X}$ Kit (Lonza, cat no. V4XP-3024) following the manufacturer's instructions and plated in media containing ROCK inhibitor Y27632 at $10 \mathrm{uM}$ for $24 \mathrm{~h}$. Cells were cultured until small to medium size colonies were formed. Then, media was supplemented with $0.5 \mu \mathrm{g} / \mathrm{mL}$ puromycin (Sigma) until reaching enough confluence to perform FACS sorting of the whole population.

\section{Fluorescence activated cell sorting}

Human iPSCs were enzymatically disaggregated and pellet was re-suspended in cold 1xPBS with 1\% BSA (Carl Roth, cat no. 8076.3) and passed through a $20 \mu \mathrm{m}$ preseparation filter (Miltenyi, cat no. 130-101-812) to ensure having a single cell suspension. Cells were sorted using FACS ARIA III (BD Bioscience), using an $85 \mu \mathrm{m}$ size nozzle and a 2.0 neural density filter. Single cell gating hierarchies on FSC and SSC width and height were applied (Additional file 1: Figure S1a) and purity mask was used. CS\&T and drop delay calibrations were ensured prior to each sorting round.

\section{Cell transfections with transposase mRNA and genotyping}

Removal of the selection module was performed by transfecting the cells with transposase PiggyBac excision-only mRNA [47] with the Stemfect RNA transfection kit (Stemgent, cat no. 00-0069) following manufacturer's instructions and the protocol described in Arias-Fuenzalida et al., 2017 [3]. Double-negative population was again purified by FACS sorting of the whole population. Correct introduction of the mutation was then evaluated via Sanger sequencing of the flanking genomic region (Fig. 1c), using primers in Additional file 6: Table S1.

\section{Microarray karyotype}

Genomic DNA from the parental (Control) and the edited $\left(\mathrm{CLN} 3{ }^{\mathrm{Q} 352 \mathrm{X}}\right.$ mutant) cell lines was isolated using GenElute Blood genomic DNA Kit (Sigma, cat no. NA2020). Samples were sent and processed at the Life \& Brain genomics facility from Bonn University, using Illumina iScan technology (Illumina). 
Evaluation of exon skipping in the $\mathrm{CLN}^{\mathrm{Q} 352 \mathrm{X}}$ mutant line Total RNA was isolated using the RNeasy Mini Kit (Qiagen, cat no. 74106) following the manufacturer's instructions. An on-column DNase digestion step was performed with RNase-Free DNase Set (Qiagen, cat no. 79254). Complementary DNA was synthesized using High Capacity RNA-to-cDNA Kit (Invitrogen, cat no. 4387406). Reverse Transcription PCR reaction was performed using GoTaq G2 Hot Start Green Master Mix (Promega, cat no. M7423) using the primers listed in Additional file 6: Table S1.

\section{Derivation and culture of endothelial cells}

Endothelial cells were differentiated from hiPS cells following a modified version of Prasain et al., 2014 protocol [64]. A density of 250.000 hiPS cells were seeded per well of a Matrigel-coated 6-well plate and cultured on Essential 8 with $1 \%$ Penicillin/Streptomycin plus 10uM ROCK inhibitor for $24 \mathrm{~h}$ (day minus three). Media was exchanged at day minus two for MEF conditioned media (R\&D Systems, cat no. AR005) supplemented with 10 ng/mL bFGF (PreproTech, cat no. 100-18B). At day minus one, media was refreshed completely with the same media and the addition of $4 \mathrm{X}$ Matrigel. Media was changed at day zero to RPMI media (Thermo, cat no. 21875-034) with the addition of 1 X B27 without vitamin A (Life Technologies, cat no. 12587-10) and $10 \mathrm{ng} / \mathrm{mL}$ Activin A (Life Technologies, cat no. PHC9564) to direct cells toward mesodermal lineage. From day one onwards, cells were cultured in RPMI with B27 media, with the addition of $10 \mathrm{ng} / \mathrm{mL}$ bFGF, $10 \mathrm{ng} / \mathrm{mL}$ BMP4 (PreproTech, cat no. $120-05$ ) and $10 \mathrm{ng} / \mathrm{mL}$ VEGF165 (PreproTech, cat no. 100-20). Cells were sustained in differentiation media conditions for two additional weeks.

\section{Generation and culture of human cerebral organoids}

Human whole brain organoids were derived from the isogenic pair hiPSCs following the Lancaster and Knoblich, 2014 protocol [44]. Cerebral organoids were maintained under differentiation conditions for 55 days after the embedding day (total 66 days).

\section{Electron microscopy}

Cultured cells and organoids were fixed using a modified Karnovsky's fixative (pH 7.4), containing 2\% PFA and 2\% glutaraldehyde in $0.1 \mathrm{M}$ sodium cacodylate buffer for $3 \mathrm{~h}$ at RT on a shaker. After this time, fixative was replaced with fresh solution and organoids were incubated for 2 days at $4{ }^{\circ} \mathrm{C}$ on a shaker. Organoids were then rinsed several times with $0.1 \mathrm{M}$ cacodylate buffer. Cultured cells were partially scraped and concentrated in a pellet then re-suspended in $2 \%$ low melting point agarose, the other part of the monolayer was flat embedded. All samples were post-fixed in $1 \%$ osmiumtetroxide, $1.5 \%$ potassiumferrocyanide in $0.1 \mathrm{M}$ cacodylate buffer and stepwise dehydrated in ethanol, including in-bloc $0.5 \%$ uranyl acetate staining during $70 \%$ ethanol incubation. Samples were embedded in epon and sectioned ultrathin at 70 $\mathrm{nm}$. Sections were collected on copper grids and stained with lead. Samples were analyzed on a Tecnai 12 electron microscope (Thermofisher Scientific, Eindhoven, The Netherlands) and representative areas were documented with a $2 \mathrm{~K}$ CCD-camera (Veleta, EMSIS, Münster, Germany). Three different blocks of each condition were sectioned and examined. Cultured endothelial cells were analysed as scraped cell sample, thus randomly orientated. Flat embedded cells were investigated in a polar manner from basolateral to apical side. Organoid samples were sectioned in three different orientations: peripheral, cross-sectioned through the centre and sectioned from the centre part of the organoids. Representative pictures are shown in the corresponding figures in the results section.

\section{Immunofluorescence in cells}

Cell fixation was done using 4\% PFA in PBS for $15 \mathrm{~min}$ at RT. Cells were washed then $3 x$ with PBS for 5 min at RT and permeabilized using $0.05 \%$ Triton-X100 in PBS for $10 \mathrm{~min}$ at $4{ }^{\circ} \mathrm{C}$. Blocking was performed for $1 \mathrm{~h}$ at RT with $10 \%$ FCS in PBS. Incubation with the corresponding primary antibodies at the required concentrations was done overnight at $4{ }^{\circ} \mathrm{C}$ in blocking buffer. For pluripotency stainings, SSEA4 (Abcam, cat no. ab16287, 1:50), Oct4 (Abcam, cat no. ab19857, 1:400), TRA-1-60 (Millipore, cat no. MAB4360, 1:50), TRA-1-81 (Millipore, cat no. MAB4381, 1:50), Nanog (Millipore, cat no. AB5731, 1:200) and hSox2 (R\&D Systems, cat no. AF2018, 1:100) were used. Endothelial cells were characterized by the expression of CD31 (Dako, cat no. M082301, 1:100) and stained for SCMAS (Abcam, cat no. ab181243, 1:100), LAMP1 (Abcam, cat no. ab25630, 1:100), GM130 (BD Bioscience, cat no. 610822, 1:200) and Rab7 (Abcam, cat no. ab50533, 1:200) for subcellular studies. Incubation with secondary antibodies was done after 5 min $3 x$ washing steps with PBS, for $2 \mathrm{~h}$ at RT in blocking buffer. All secondary antibodies (Invitrogen) were conjugated to Alexa Fluor fluorochromes and used in a 1:1000 dilution together with a nuclei counterstain by Hoechst 33342 (Life Technologies, cat no. H21492). Cells were washed $3 x$ with PBS, and either mounted with Fluoromount-G mounting medium (Southern Biotech, cat no. 0100-01) or covered with PBS and imaged directly after.

\section{Immunofluorescence in organoid sections}

Organoids were fixed with $4 \%$ PFA overnight on a shaker at $4{ }^{\circ} \mathrm{C}$ and washed $3 \mathrm{x}$ with PBS. Afterwards, they were embedded in $3 \%$ low-melting point agarose 
(Biozym, cat no. 840100) in PBS and incubated at RT until the agarose solidified. The agarose block was either covered with PBS and kept at $4{ }^{\circ} \mathrm{C}$ or sectioned immediately. If not indicated otherwise, $80 \mu \mathrm{m}$ sections were cut using a vibratome (Leica VT1000s), set to a speed of 6.1 and a frequency of 9. Sections were permeabilized and blocked in one-step with PBS containing $0.1 \%$ sodium azide, $0.1 \%$ sodium citrate, $5 \%$ goat or donkey serum and $2 \% \mathrm{BSA}$, for $90 \mathrm{~min}$ at $\mathrm{RT}$ on a shaker. Incubation with the corresponding primary antibodies at the required concentration was performed in blocking buffer containing $0.1 \%$ Triton-X for $48 \mathrm{~h}$ at $4{ }^{\circ} \mathrm{C}$. Synaptophysin (Abcam, cat no. ab8049, 1:50), PSD95 (Invitrogen, cat no. 51-6900, 1:300), vGAT (Sigma, cat no. HPA058859, 1:200), TUJ1 (Millipore, Cat no. AB9354) and MAP 2 (Abcam, cat no. ab32454, 1:100) antibodies were used to evaluate synaptic markers. GFAP (Millipore, cat.no. AB5541, 1:1000) and MAP 2 (Abcam, cat no. ab32454, 1:100) antibodies were used for astrocytosis assessment. CTIP2 (Abcam, cat no. ab18465, 1:100), SATB2 (Abcam, cat no. 34735, 1:100), TBR1 (Abcam, cat no. 31940, 1: 300) and FOXG1 (Abcam, cat no. 18259, 1:1000) antibodies were used for brain organoid characterization. SOX2 (Abcam, cat no. ab97959, 1:200) and Nestin (BD Bioscience, cat no. 611659, 1:600) primary antibodies were used for neural stem cell evaluation. After 3x wash with PBS for $15 \mathrm{~min}$ at RT, secondary antibodies were added in a solution of PBS with $0.05 \%$ Tween-20. All secondary antibodies (Invitrogen) were conjugated to Alexa Fluor fluorochromes and used in a 1:1000 dilution together with a nuclei counterstain by Hoechst 33342 (Life Technologies, cat no. H21492). Incubation was done for $2 \mathrm{~h}$ at $\mathrm{RT}$ on a shaker and protected from light. Sections were washed $3 \mathrm{x}$ with $0.05 \%$ Tween-20 in PBS for $5 \mathrm{~min}$ at RT and $1 \mathrm{x}$ with water and then mounted with Fluoromount-G mounting medium (Southern Biotech, cat no. 0100-01) on a glass slide. Sections were dried overnight at RT and in the dark.

\section{Western blotting}

Cerebral organoids were individually lysed in a Urea containing lysis buffer (7 M Urea, $2 \mathrm{M}$ Thiourea, 2\% CHAPS and $1 \mathrm{M}$ DTT) containing $1 \mathrm{X}$ protease inhibitor cocktail (Sigma). Protein samples were resolved by denaturing SDS polyacrylamide gel electrophoresis (SDS-PAGE) in 15\% polyacrylamide gels. Proteins were transferred from the gel to PVDF membranes in an iBlot2 device (Thermo). Membranes were incubated with SuperSignal West Pico Chemiluminescent Substrate (Thermo, cat no. \#34580). Enhanced chemiluminiscent signal was detected in a STELLA imaging system. Evaluated antibodies were TPP1 (Abcam, cat no. ab96690, 1:1000), SCMAS (Abcam, cat no. ab181243, 1: 1000), RIP1 (BD Bioscience, cat no. 610459, 1:1000), RIP3 (Abcam, cat no. ab152130, 1:1000), FOXG1 (Abcam, cat no. 18259, 1:500), SATB2 (Abcam, cat no. 34735, 1:250) and GAPDH (Abcam, cat no. 9485, 1:1000).

\section{Human Cathepsin D ELISA detection}

Cathepsin D levels were evaluated using the Human Cathepsin D SimpleStep ELISA kit (Abcam, cat no. ab213470) following the manufacturer's instructions. Briefly, cerebral organoids were individually disrupted in $200 \mu \mathrm{L}$ of chilled 1X PTR cell extraction buffer and incubated for $20 \mathrm{~min}$ on ice, while homogenizing with either a pipette tip or needles. Samples and standards were loaded into the 96-well assay plate strips and incubated for $1 \mathrm{~h}$ at RT together with the antibody cocktail. Wells were washed $3 \mathrm{x}$ in $1 \mathrm{x}$ PT wash buffer and incubated with $\mathrm{TMB}$ substrate for $10 \mathrm{~min}$. Reaction was the stopped and optical density at $405 \mathrm{~nm}$ measured in a Cytation 5 plate reader (BioTek) as an endpoint measurement. For normalization, sample protein concentration was quantified using Protein Quantification Assay (Macherey-Nagel, cat no. 740967.250).

\section{Terminal deoxynucleotidyl transferase biotin-dUTP nick end labeling (TUNEL) assay}

General apoptosis within the organoids was detected using the In situ cell death detection kit, TMR red (Merck, cat no. 12156792910) following the manufacturer's instructions, adapted to screen organoid sections. Briefly, organoid sections were permeabilized with $0.1 \%$ Triton- $\mathrm{X}$ in $0.1 \%$ sodium citrate for $8 \mathrm{~min}$ at $4{ }^{\circ} \mathrm{C}$. After $3 \mathrm{x}$ wash with $\mathrm{PBS}$, sections were incubated with the TUNEL reaction mixture for $1 \mathrm{~h}$ at $37^{\circ} \mathrm{C}$ and in a humidified atmosphere and in the dark. Hoechst 33342 (Life Technologies, cat no. H21492, 1:1000) was added to the mix to visualize the nuclei. Positive controls were treated in a previous step with $5 \mathrm{uL}$ of Deoxyribonuclease I (DNase I) (Sigma-Aldrich, cat no. AMPD1-1KT, 1unit/ $\mathrm{uL}$ ) for $10 \mathrm{~min}$ at RT to induce DNA strand breaks. Negative controls were incubated with label solution only. Following $3 \mathrm{x}$ wash with $\mathrm{PBS}$, sections were mounted with Fluoromount-G mounting medium (Southern Biotech, cat no. 0100-01) on a glass slide. Sections were dried overnight at RT and in the dark. Imaging was performed in Operetta High-content Imaging System (Perkin Elmer) in a range of $520-560 \mathrm{~nm}$ excitation and 570-620 $\mathrm{nm}$ emission.

\section{RNA-Seq}

Total RNA was isolated from cerebral organoids using the RNeasy Mini Kit (Qiagen, cat no. 74106). Six replicates per condition, constituted by a pool of three organoids per replicate, were extracted. RNA quality was assessed in a Bioanalyzer 2100 (Agilent). In a second step, library preparation for sequencing was done with $1 \mu \mathrm{g}$ of total RNA using the TruSeq mRNA Stranded 
Library Prep Kit (Illumina) according to manufacturer's protocol. Pooled libraries were sequenced on NextSeq500 using manufacturer's instructions.

\section{RNA-Seq data processing and analysis}

Illumina $\mathrm{Hi}$-Seq single-end reads were filtered by using BBDuk $($ trimq $=10$ qtrim $=\mathrm{r}$ ktrim $=\mathrm{r} \mathrm{k}=23$ mink $=11$ hdist $=1$ tpetbominlen $=40$; http://jgi.doe.gov/data-andtools/bb-tools/) to remove illumina adapters, PhiX library adapters, and to quality trim the reads. FastQC [2] was used to check the quality of the reads in order to assure that only high-quality reads are retained for subsequent analysis. Resulting reads were mapped to human GRCh37 genome by using tophat (version 2.1.1) [75] (library-type $=$ fr-secondstrand) and Bowtie 2 (version 2.3.2.0). Obtained alignment files were sorted by using samtools (version 1.6-5) [46] and the statistics of the alignment rate were obtained by using samtoolsflagstat. Cufflinks (version 2.2.1) [75] was used to quantify the transcripts and resulting expression values per gene were obtained in FPKM (fragments per kb per million reads). Differential expression analysis between the Control and $\mathrm{CLN}_{3}{ }^{\mathrm{Q} 352 \mathrm{X}}$ mutant samples was conducted by using the cuffdiff program from the cufflinks tool. Only significantly, differentially expressed genes with an absolute $\log 2$ fold change greater than 1 were considered for subsequent analysis.

\section{Gene regulatory network reconstruction}

For the set of significantly differentially expressed genes (DEGs), the experimentally validated direct gene-gene interactions were retrieved from MetaCore (Clarivate Analytics). The interaction types belonging to categories "Transcription regulation" and "Binding" were kept in the prior knowledge network (PKN) from MetaCore. Differential network inference method proposed by [84] was used to prune the network edges (interactions) which are not compatible with the discretized gene expression program of the respective phenotype. Briefly, this method uses discretized differential gene expression data and infers two networks representing the mutant (disease) and cotnrol (healthy) phenotypes as steady states. Some of the interactions derived from MetaCore have an unspecified regulatory effect, as the exact mechanism of regulation is not known in those cases. The proposed algorithm also infers the regulatory effect (activation or inhibition) for such unspecified interactions based on the given gene expression pattern.

\section{Identification of network perturbation candidates}

The differential network topology allowed us to identify common and phenotype-specific positive and negative elementary circuits, i.e. a network path which starts and ends at the same node with all the intermediate nodes being traversed only once. These circuits have been shown to play a significant role in maintaining network stability [27] and the existence of these circuits is considered to be a necessary condition for having a stable steady (network) state [74]. Considering the importance of these circuits, it has been shown that perturbation of genes in the positive circuits induces a phenotypic transition [19]. Furthermore, the differential network topology also aids in identifying the differential regulators of the genes, which are common to both phenotype-specific networks. Altogether, the differential regulators and genes in the elementary circuits constitute an optimal set of candidate genes for network perturbation as they are able to revert most of the gene expression program upon perturbation. Identification of network perturbation candidates was carried out by using the Java implementation proposed by Zickenrott and colleagues [84].

\section{In silico network simulation analysis for phenotype reversion}

The Java implementation from Zickenrott and colleagues [84] was used to perform the network simulation analysis by perturbing multi-target combinations of up to four candidate genes identified in the previous step. The used algorithm gives a ranked list of single- and multigene(s) combinations (4 genes maximally) and their scores, which represent the number of genes whose expression is being reverted upon inducing the chosen perturbation in the diseased network. If a single- or multi-gene(s) perturbation combination obtains a high score, it is indicative of its ability to regulate the expression of a large number of downstream genes, hence playing a crucial role in the maintenance and stability of the phenotype under consideration.

\section{Gene and pathway enrichment analyses}

MetaCore (Clarivate Analytics) and EnrichNet [23] were used to conduct the gene ontology (GO) and pathway enrichment analysis. The set of upregulated genes in the diseased network were used to identify the most overrepresented biological processes and molecular functions associated with the genes in the network. Similarly, the most enriched biological pathways associated with genes that are upregulated in the pathologic network phenotype were described. The same kind of analysis was conducted to show downregulations in biological processes and pathways.

\section{Metabolomics}

Metabolites from cerebral organoids were extracted and subsequently prepared for non-targeted gas chromatographymass spectrometry (GC-MS) measurements, as previously 
described [34]. Five replicates per condition, constituted by a pool of three organoids per replicate, were extracted. Organoids were homogenized using a bead mill homogenizer system (Precellys24) and ceramic beads to produce a homogeneous suspension. Metabolites were then extracted with water/methanol/chloroform, yielding a three-phase system, where polar metabolites are enriched in the upper phase, non-polar metabolites in the lower phase and cell debris form a solid interphase. Polar phase was transferred into a sample vial and solvents were evaporated in a rotary vacuum evaporator at $-4{ }^{\circ} \mathrm{C}$ until dry and stored at $-80^{\circ} \mathrm{C}$ until GC-MS analysis. Subsequent metabolic profiling is performed with gas chromatography coupled to mass spectrometry on an Agilent 7890B GC - Agilent 5977A MSD system. Post processing of the data was performed with the MetaboliteDetector software (http://md.tu-bs.de). Data was then manually curated, removing any possible contamination of external compounds due to sample preparation and the internal standards.

\section{Image acquisition}

Brightfield and fluorescence representative images of the gene editing procedure, cell culture characterization and organoid images for area measurements were acquired using an inverted microscope (Zeiss Axio ObserverZ1). Synapse and autofluorescence analyses were performed on organoid sections imaged on a confocal (Zeiss LSM 710) laser-scanning microscope. Sections were usually imaged at $20-x$ and either tile scanning of the whole organoid sections were acquired or random regions of interest (ROI) within the sections were selected. Images were further processed with Zen Software (Zeiss) and quantifications were performed on ImageJ $(\mathrm{NIH})$. The high content microscope OPERA QEHS spinning disk microscope (Perkin Elmer) was used for 2D endothelial cell imaging, using a 63-x water immersion objective. On the other hand, Operetta High-content Imaging System (Perkin Elmer) was used to acquire volumetric images (Z-stacking) of the entire organoid sections (Tile-scanning). The $20-\mathrm{x}$ objective was used for this purpose. In both cases, volumetric images of plated cells and 3D images of cerebral organoids e.g. TUNEL assay- were analyzed in Matlab (Version 2017b, The MathWorks Inc.), with in-house developed image analysis algorithms which automate the segmentation of nuclei and allow structure-specific feature extraction. The expression level of any marker was expressed as positive pixel of the marker, and commonly normalized by the pixel count of Hoechst.
Statistical analysis and graphical representation All the statistical analysis were performed in GraphPad Prism (Version 6.01). Significance asterisks represent $P<0.05^{*}, P<0.01^{* *}, P<0.001^{* * *}, P<0.0001^{* * * * ; n s}$ stands for not significant.

\section{Supplementary information}

Supplementary information accompanies this paper at https://doi.org/10. 1186/s40478-019-0871-7.

Additional file 1: Figure S1. Characterization of the CLN3 isogenic pair. Additional file 2: Figure S2. Ultrastructural evaluation of different organelles in endothelial cells.

Additional file 3: Figure S3. Characterization of hiPSC-derived cerebral organoids.

Additional file 4: Figure S4. Gene regulatory networks.

Additional file 5: Figure S5. Gene expression levels of relevant proteins.

Additional file 6: Table S1. Oligonucleotides used in this study. (*) This sgRNA was used for the editing. Table S2. Off-target analysis. Table S3. Identifiable metabolite changes detected by unsupervised ANOVA hierarchical clustering. Table S4. List of significant $(p<0.05)$ metabolic changes detected by Mann-Whitney test between genotypes. Table S5. Top 10 key candidate genes from single- and multi-gene network perturbation simulation analysis.

\section{Acknowledgements}

J. C. S.'s lab is supported by Fonds National de la Recherche (FNR) Luxembourg (Proof of Concept, FNR/PoC16/11559169). We would like to thank the LCSB bio-imaging platform for flow cytometry support and the stem cells, gene editing and organoid platform for support. Zdenka Hodak of the LCSB Metabolomics Platform for providing technical assistance. NCLStiftung and Batten Research Fonds (BRF) supported G.GG. J.J. was supported by FNR (AFR, Aides à la Formation-Recherche).

\section{Authors' contributions}

JCS conceived the study. GG-G, JA-F and JCS designed experiments. GG-G, JA-F, JJ, DZ, NP, RH, and CJ performed experiments. GG-G, JA-F, JJ, DZ, MA, NP and SB performed data analysis. WFEK and PH provided patient material. $H Z, A S, H P, H R S$ and JCS supervised the study. GG-G, MA and JCS wrote the manuscript and organized display items. All the authors revised the manuscript and agreed to the final version of the manuscript.

\section{Availability of data and materials}

All original and processed data used in this study are publicly available at this doi: https://doi.org/10.17881/lcsb.2019130913.01.

\section{Competing interests}

The authors declare that they have no competing interests.

\section{Author details}

'Luxembourg Centre for Systems Biomedicine (LCSB), Laboratory of Developmental and Cellular Biology, University of Luxembourg, Avenue du Swing 6, Belvaux, Luxembourg. 'Laboratory of Cellular and Developmental Biologie, Max Planck Institute for Molecular Biomedicine, Röntgenstrasse, Münster, Germany. ${ }^{3}$ Electron Microscopy Unit, Max Planck Institute for Molecular Biomedicine, Röntgenstrasse, Münster, Germany. ${ }^{4}$ Luxembourg Centre for Systems Biomedicine (LCSB), Laboratory of Computational Biology, University of Luxembourg, Avenue du Swing 6, Belvaux, Luxembourg. ${ }^{5}$ Department of Psychiatry and Neuropsychology, School for Mental Health and Neuroscience (MHeNS), Maastricht University, Maastricht, the Netherlands. 'Luxembourg Centre for Systems Biomedicine (LCSB), Laboratory of Eco-Systems Biology, University of Luxembourg, Avenue des Hauts Fourneaux 7, Esch-sur-Alzette, Luxembourg. ${ }^{7}$ Luxembourg Centre for Systems Biomedicine (LCSB), Laboratory of Enzymology and Metabolism, University of Luxembourg, Avenue du Swing 6, Esch-sur-Alzette, 
Luxembourg. ${ }^{8}$ Department of Metabolic Diseases, Wilhelmina Children's Hospital, University Medical Center Utrecht, Utrecht, the Netherlands. ${ }^{9}$ Ruhr-University Bochum, Medical Faculty, Department of Anatomy and Molecular Embryology, Bochum, Germany. ${ }^{10}$ IKERBASQUE, Basque Foundation for Science, Bilbao, Spain. ${ }^{11} \mathrm{ClC}$ bioGUNE, Bizkaia Technology Park, 801 Building, Derio, Spain. ${ }^{12}$ NCL Foundation, Holstenwall 10, Hamburg, Germany.

\section{Received: 22 September 2019 Accepted: 13 December 2019} Published online: 30 December 2019

\section{References}

1. Allende ML, Cook EK, Larman BC, Nugent A, Brady JM, Golebiowski D et al (2018) Cerebral organoids derived from Sandhoff disease-induced pluripotent stem cells exhibit impaired neurodifferentiation. J Lipid Res 59: 550-563

2. Andrews S (2010) FastQC: a quality control tool for high throughput sequence data. Available online at http://www.bioinformatics.babraham.ac uk/projects/fastqc

3. Arias-Fuenzalida J, Jarazo J, Qing X, Walter J, Gomez-Giro G, Nickels SL et al (2017) FACS-assisted CRISPR-Cas9 genome editing facilitates Parkinson's disease modeling. Stem Cell Rep 9:1423-1431

4. Barrangou R, Doudna JA (2016) Applications of CRISPR technologies in research and beyond. Nat Biotechnol 34:933

5. Baumuratov AS, Antony PMA, Ostaszewski M, He F, Salamanca L, Antunes L et al (2016) Enteric neurons from Parkinson's disease patients display ex vivo aberrations in mitochondrial structure. Sci Rep 6:33117

6. Bennett MJ, Rakheja D (2013) The neuronal ceroid-lipofuscinoses. Dev Disabil Res Rev 17:254-259

7. Berger SB, Bertin J, Gough PJ (2016) Life after death: RIP1 and RIP3 move beyond necroptosis. Cell Death Dis 2:16056

8. Bosch ME, Kielian T (2018) Astrocytes in juvenile neuronal ceroid lipofuscinosis (CLN3) display metabolic and calcium signaling abnormalities. J Neurochem 148:612-624

9. Bozorg S, Ramirez-Montealegre D, Chung M, Pearce DA (2009) Juvenile neuronal ceroid lipofuscinosis (JNCL) and the eye. Surv Ophthalmol 54:463-471

10. Brennand KJ (2017) Personalized medicine in a dish: the growing possibility of neuropsychiatric disease drug discovery tailored to patient genetic variants using stem cells. Stem Cell Investig 4:91

11. Britanova O, de Juan RC, Cheung A, Kwan KY, Schwark M, Gyorgy A et al (2008) Satb2 is a postmitotic determinant for upper-layer neuron specification in the neocortex. Neuron 57:378-392

12. Brooks DA, Muller VJ, Hopwood JJ (2006) Stop-codon read-through for patients affected by a lysosomal storage disorder. Trends Mol Med 12:367-373

13. Burkovetskaya M, Karpuk N, Kielian T (2017) Age-dependent alterations in neuronal activity in the hippocampus and visual cortex in a mouse model of juvenile neuronal Ceroid Lipofuscinosis (CLN3). Neurobiol Dis 100:19-29

14. Calvano SE, Xiao W, Richards DR, Felciano RM, Baker HV, Cho RJ et al (2005) A network-based analysis of systemic inflammation in humans. Nature 437: 1032-1037

15. Cantalupo G, Alifano P, Roberti V, Bruni CB, Bucci C (2001) Rab-interacting lysosomal protein (RILP): the Rab7 effector required for transport to lysosomes. EMBO J 20:683-693

16. Cárcel-Trullols J, Kovács AD, Pearce DA (2017) Role of the Lysosomal membrane protein, $\mathrm{CLN} 3$, in the regulation of Cathepsin D activity. J Cell Biochem 118:3883-3890

17. Cartegni L, Chew SL, Krainer AR (2002) Listening to silence and understanding nonsense: exonic mutations that affect splicing. Nat Rev Genet 3:285-298

18. Chandrachud U, Walker MW, Simas AM, Heetveld S, Petcherski A, Klein M et al (2015) Unbiased cell-based screening in a neuronal cell model of batten disease highlights an interaction between Ca2+ homeostasis, autophagy, and CLN3 protein function. J Biol Chem 290:14361-14380

19. Crespo I, Perumal TM, Jurkowski W, del Sol A (2013) Detecting cellular reprogramming determinants by differential stability analysis of gene regulatory networks. BMC Syst Biol 7:140

20. Doench JG, Hartenian E, Graham DB, Tothova Z, Hegde M, Smith I et al (2014) Rational design of highly active sgRNAs for CRISPR-Cas9-mediated gene inactivation. Nat Biotechnol 32:1262-1267

21. Dwyer ND, O'Leary DD (2001) Tbr1 conducts the orchestration of early cortical development. Neuron 29:309-311
22. Eckler MJ, Chen B (2014) Fez family transcription factors: controlling neurogenesis and cell fate in the developing mammalian nervous system. BioEssays 36:788-797

23. Glaab E, Baudot A, Krasnogor N, Schneider R, Valencia A (2012) EnrichNet: network-based gene set enrichment analysis. Bioinformatics 28:i451-i457

24. Glantz LA, Gilmore JH, Hamer RM, Lieberman JA, Jarskog LF (2007) Synaptophysin and postsynaptic density protein 95 in the human prefrontal cortex from mid-gestation into early adulthood. Neuroscience 149:582-591

25. Golabek AA, Kida E (2006) Tripeptidyl-peptidase I in health and disease. Biol Chem 387:1091-1099

26. Golabek AA, Kida E, Walus M, Kaczmarski W, Michalewski M, Wisniewski KE (2000) CLN3 protein regulates lysosomal pH and alters intracellular processing of Alzheimer's amyloid-beta protein precursor and cathepsin D in human cells. Mol Genet Metab 70:203-213

27. Gouzé JL (1998) Positive and negative circuits in dynamical systems. J Biol Syst 06:11-15

28. Grünewald B, Lange MD, Werner C, O'Leary A, Weishaupt A, Popp S et al (2017) Defective synaptic transmission causes disease signs in a mouse model of juvenile neuronal ceroid lipofuscinosis. Elife 14:6

29. Guerra F, Bucci C (2016) Multiple Roles of the Small GTPase Rab7. Cells 5:3

30. Hanashima C, Li SC, Shen L, Lai E, Fishell G (2004) Foxg1 suppresses early cortical cell fate. Science 303:56-59

31. Hersrud SL, Kovács AD, Pearce DA (2016) Antigen presenting cell abnormalities in the $\mathrm{Cln} 3(-/-)$ mouse model of juvenile neuronal ceroid lipofuscinosis. Biochim Biophys Acta 1862:1324-1336

32. Huber RJ, Mathavarajah S (2019) Comparative transcriptomics reveals mechanisms underlying cln3-deficiency phenotypes in Dictyostelium. Cell Signal 58:79-90

33. International Batten Disease Consortium (1995) Isolation of a novel gene underlying batten disease, CLN3. Cell 82:949-957

34. Jäger C, Hiller K, Buttini M (2016) Metabolic profiling and quantification of neurotransmitters in mouse brain by gas chromatography-mass spectrometry. Curr Protoc Mouse Biol 6:333-342

35. Jarazo J, Qing X, Schamborn JC (2019) Guidelines for fluorescent guided biallelic HDR tageting selection with piggyback system removal for gene editing. Front Genet 10:190

36. Kerschnitzki M, Kollmannsberger P, Burghammer M, Duda GN, Weinkamer R, Wagermaier W et al (2013) Architecture of the osteocyte network correlates with bone material quality. J Bone Miner Res 28:1837-1845

37. Kielar C, Wishart TM, Palmer A, Dihanich S, Wong AM, Macauley SL et al (2009) Molecular correlates of axonal and synaptic pathology in mouse models of batten disease. Hum Mol Genet 18:4066-4080

38. Kohlschütter A, Laabs R, Albani M (1988) Juvenile neuronal ceroid lipofuscinosis (JNCL): quantitative description of its clinical variability. Acta Paediatr Scand 77:867-872

39. Koike M, Nakanishi H, Saftig P, Ezaki J, Isahara K, Ohsawa Y et al (2000) Cathepsin D deficiency induces lysosomal storage with ceroid lipofuscin in mouse CNS neurons. J Neurosci 20:6898-6906

40. Kori M, Aydın B, Unal S, Arga KY, Kazan D (2016) Metabolic biomarkers and Neurodegeneration: a pathway enrichment analysis of Alzheimer's disease, Parkinson's disease, and amyotrophic lateral sclerosis. OMICS 20:645-661

41. Kousi M, Lehesjoki AE, Mole SE (2012) Update of the mutation spectrum and clinical correlations of over 360 mutations in eight genes that underlie the neuronal ceroid lipofuscinoses. Hum Mutat 33:42-63

42. Kuper WFE, van Alfen C, Rigterink RH, Fuchs SA, van Genderen MM, van Hasselt PM (2018) Timing of cognitive decline in CLN3 disease. J Inherit Metab Dis 41:257-261

43. Kwon JM, Adams H, Rothberg PG, Augustine EF, Marshall FJ, Deblieck EA et al (2011) Quantifying physical decline in juvenile neuronal ceroid lipofuscinosis (Batten disease). Neurology 77:1801-1807

44. Lancaster MA, Knoblich JA (2014) Generation of cerebral organoids from human pluripotent stem cells. Nat Protoc 9:2329-2340

45. Lancaster MA, Renner M, Martin CA, Wenzel D, Bicknell LS, Hurles ME et al (2013) Cerebral organoids model human brain development and microcephaly. Nature 501:373-379

46. Li H, Handsaker B, Wysoker A, Fennell T, Ruan J, Homer N et al (2009) The sequence alignment/map format and SAMtools. Bioinformatics 25: 2078-2079

47. Li X, Burnight ER, Cooney AL, Malani N, Brady T, Sander JD et al (2013b) piggyBac transposase tools for genome engineering. Proc Natl Acad Sci U S A 110:E2279-E2287 
48. Llavero Hurtado M, Fuller HR, Wong AMS, Eaton SL, Gillingwater TH, Pennetta G et al (2017) Proteomic mapping of differentially vulnerable presynaptic populations identifies regulators of neuronal stability in vivo. Sci Rep 7:12412

49. Lojewski X, Staropoli JF, Biswas-Legrand S, Simas AM, Haliw L, Selig MK et al (2014) Human iPSC models of neuronal ceroid lipofuscinosis capture distinct effects of TPP1 and CLN3 mutations on the endocytic pathway. Hum Mol Genet 23:2005-2022

50. March PA, Wurzelmann S, Walkley SU (1995) Morphological alterations in neocortical and cerebellar GABAergic neurons in a canine model of juvenile batten disease. Am J Med Genet 57:204-212

51. Mariani J, Simonini MV, Palejev D, Tomasini L, Coppola G, Szekely AM et al (2012) Modeling human cortical development in vitro using induced pluripotent stem cells. Proc Natl Acad Sci U S A 109:12770-12775

52. Marmorstein AD, Marmorstein LY, Sakaguchi H, Hollyfield JG (2002) Spectral profiling of autofluorescence associated with lipofuscin, Bruch's membrane, and sub-RPE deposits in normal and AMD eyes. Investig Ophthalmol Vis Sci 43:2435-2441

53. Mitchison HM, Lim MJ, Cooper JD (2004) Selectivity and types of cell death in the neuronal ceroid lipofuscinoses. Brain Pathol 14:86-96

54. Mole SE, Williams RE, Goebel HH (2011) The Neuronal Ceroid Lipofuscinoses (Batten Disease). In: Contemporary Neurology Series, 2nd edn. Oxford University Press, Oxford, pp 361-365

55. Munroe PB, Mitchison HM, O'Rawe AM, Anderson JW, Boustany RM, Lerner TJ et al (1997) Spectrum of mutations in the batten disease gene, CLN3. Am J Hum Genet 61:310-316

56. Nikouei K, Muñoz-Manchado AB, Hjerling-Leffler J (2016) BCL11B/CTIP2 is highly expressed in GABAergic interneurons of the mouse somatosensory cortex. J Chem Neuroanat 71:1-5

57. Olson JM, Asakura A, Snider L, Hawkes R, Strand A, Stoeck J et al (2001) NeuroD2 is necessary for development and survival of central nervous system neurons. Dev Biol 234:174-187

58. Osório NS, Sampaio-Marques B, Chan CH, Oliveira P, Pearce DA, Sousa N et al (2009) Neurodevelopmental delay in the CIn3Deltaex7/8 mouse model for batten disease. Genes Brain Behav 8:337-345

59. Ouseph MM, Kleinman ME, Wang QJ (2016) Vision loss in juvenile neuronal ceroid lipofuscinosis (CLN3 disease). Ann N Y Acad Sci 1371:55-67

60. Palmer DN, Fearnley IM, Walker JE, Hall NA, Lake BD, Wolfe LS et al (1992) Mitochondrial ATP synthase subunit c storage in the ceroid-lipofuscinoses (batten disease). Am J Med Genet 42:561-567

61. Parviainen L, Dihanich S, Anderson GW, Wong AM, Brooks HR, Abeti R et al (2017) Glial cells are functionally impaired in juvenile neuronal ceroid lipofuscinosis and detrimental to neurons. Acta Neuropathol Com 5:74

62. Pérez-Poyato MS, Milà Recansens M, Ferrer Abizanda I, Montero Sánchez R, Rodríguez-Revenga L, Cusí Sánchez V (2011) Juvenile neuronal ceroid lipofuscinosis: clinical course and genetic studies in Spanish patients. Inherit Metab Dis 34:1083-1093

63. Pontikis CC, Cella CV, Parihar N, Lim MJ, Chakrabarti S, Mitchison HM et al (2004) Late onset neurodegeneration in the $C \ln 3-/-$ mouse model of juvenile neuronal ceroid lipofuscinosis is preceded by low level glial activation. Brain Res 1023:231-242

64. Prasain N, Lee MR, Vemula S, Meador JL, Yoshimoto M, Ferkowicz MJ et al (2014) Differentiation of human pluripotent stem cells to cells similar to cord-blood endothelial colony-forming cells. Nat Biotechnol 32:1151-1157

65. Pujana MA, Han JD, Starita LM, Stevens KN, Tewari M, Ahn JS et al (2007) Network modeling links breast cancer susceptibility and centrosome dysfunction. Nat Genet 39:1338-1349

66. Qian X, Nguyen HN, Song MM, Hadiono C, Ogden SC, Hammack C et al (2016) Brain-region-specific Organoids using mini-bioreactors for modeling ZIKV exposure. Cell 165:1238-1254

67. Ran FA, Hsu PD, Wright J, Agarwala V, Scott DA, Zhang F (2013) Genome engineering using the CRISPR-Cas9 system. Nat Protoc 8:2281-2308

68. Reinhardt P, Glatza M, Hemmer K, Tsytsyura Y, Thiel CS, Hoing S et al (2013) Derivation and expansion using only small molecules of human neural progenitors for neurodegenerative disease modeling. PLoS One 8:e59252

69. Renner M, Lancaster MA, Bian S, Choi H, Ku T, Peer A et al (2017) Selforganized developmental patterning and differentiation in cerebral organoids. EMBO J 36:1316-1329

70. Santavuori P, Vanhanen SL, Autti T (2001) Clinical and neuroradiological diagnostic aspects of neuronal ceroid Lipofuscinoses disorders. Eur J Paediatr Neurol 5(Suppl A):157-161
71. Schulz A, Kohlschütter A, Mink J, Simonati A, Williams R (2013) NCL diseasesclinical perspectives. Biochim Biophys Acta 1832:1801-1806

72. Steinhart Z, Angers S (2018) Wnt signaling in development and tissue homeostasis. Development 145:11

73. Stemmer M, Thumberger T, del Sol KM, Wittbrodt J, Mateo JL (2017) CCTop: an intuitive, flexible and reliable CRISPR/Cas9 target prediction tool. PLoS One 12:e0176619

74. Thomas R (1981) On the Relation Between the Logical Structure of Systems and Their Ability to Generate Multiple Steady States or Sustained Oscillations. In: Della Dora J, Demongeot J, Lacolle B (eds) Numerical Methods in the Study of Critical Phenomena. Springer Series in Synergetics, vol 9. Springer, Berlin, Heidelberg

75. Trapnell C, Roberts A, Goff L, Pertea G, Kim D, Kelley DR et al (2012) Differential gene and transcript expression analysis of RNA-seq experiments with TopHat and cufflinks. Nat Protoc 7:562-578

76. Uvebrant P, Hagberg B (1997) Neuronal ceroid lipofuscinoses in Scandinavia. Epidemiology and clinical pictures. Neuropediatrics 28:6-8

77. Vesa J, Chin MH, Oelgeschläger K, Isosomppi J, DellAngelica EC, Jalanko A et al (2002) Neuronal ceroid lipofuscinoses are connected at molecular level: interaction of CLN5 protein with CLN2 and CLN3. Mol Biol Cell 13:2410-2420

78. Wager K, Zdebik AA, Fu S, Cooper JD, Harvey RJ, Russell C (2016) Neurodegeneration and epilepsy in a Zebrafish model of CLN3 disease (batten disease). PLoS One 11:e0157365

79. Wang F, Wang H, Tuan HF, Nguyen DH, Sun V, Keser V et al (2014) Next generation sequencing-based molecular diagnosis of retinitis pigmentosa: identification of a novel genotype-phenotype correlation and clinical refinements. Hum Genet 133:331-345

80. Wang RN, Green J, Wang Z, Deng Y, Qiao M, Peabody M et al (2014) Bone morphogenetic protein (BMP) signaling in development and human diseases. Genes Dis 1:87-105

81. Williams R (2011) NCL incidence and prevalence data. In: Mole SE, Williams RE, Goebel HH (eds) The neuronal Ceroid Lipofuscinoses (batten disease), Contemporary Neurology Series, 2nd edn. Oxford University Press, Oxford, pp 361-365

82. Wu MY, Hill CS (2009) Tgf-beta superfamily signaling in embryonic development and homeostasis. Dev Cell 16:329-343

83. Zhang X, Zhang D, Chen SC, Lamey T, Thompson JA, McLaren T et al (2018) Generation of an induced pluripotent stem cell line from a patient with non-syndromic CLN3-associated retinal degeneration and a coisogenic control line. Stem Cell Res 29:245-249

84. Zickenrott S, Angarica VE, Upadhyaya BB, del Sol A (2016) Prediction of disease-gene-drug relationships following a differential network analysis. Cell Death Dis 7:e2040

\section{Publisher's Note}

Springer Nature remains neutral with regard to jurisdictional claims in published maps and institutional affiliations.

Ready to submit your research? Choose BMC and benefit from:

- fast, convenient online submission

- thorough peer review by experienced researchers in your field

- rapid publication on acceptance

- support for research data, including large and complex data types

- gold Open Access which fosters wider collaboration and increased citations

- maximum visibility for your research: over $100 \mathrm{M}$ website views per year

At BMC, research is always in progress.

Learn more biomedcentral.com/submission 